\title{
TYPE MATERIAL OF TAXA DESCRIBED BY COUSIN AND JOUSSEAUME IN THE ROYAL BELGIAN INSTITUTE OF NATURAL SCIENCES, BRUSSELS
}

\author{
ABRAHAM S. H. BREURE ${ }^{1,2}$ \\ ${ }^{1}$ Royal Belgian Institute of Natural Science, Vautierstraat 29, B-1000 Brussels, Belgium \\ (ashbreure@protonmail.com); (1) https://orcid.org/0000-0001-9357-0501 \\ ${ }^{2}$ Natural History Museum, Department of Life Science, Invertebrate Division, London SW7 5BD, \\ United Kingdom
}

ABSTRACT: Type material is documented for 28 taxa described by Cousin and Jousseaume on the basis of material collected by Cousin in Ecuador. These taxa belong to 12 families (Achatinidae, Ampullariidae, Bulimulidae, Labyrinthidae, Lymnaeidae, Neocyclotidae, Orthalicidae, Planorbidae, Proserpinellidae, Scolodontidae, Solaropsidae, Succineidae). Type specimens and their labels are figured. In some instances, the specimen labels provide more precise locality information than the literature reference. Isomeria bourcieri var. lutea Cousin, 1887 is now considered a junior objective synonym of Isomeria bourcieri (L. Pfeiffer, 1853).

KEY WORDS: Ecuador, taxa list, Mollusca, Gastropoda

\section{INTRODUCTION}

Auguste Édouard Cousin (1835-1899) was the French consul in Ecuador for many years. He published only a single paper (COUSIN 1887), but appears to have had a quite substantial collection of both marine and non-marine Mollusca from all over the world. An inventory of this collection was found in 2010 while researching Orthalicoidea type material in the Brussels museum (BREURE 2011: 3-4, fig. 2C). Although the title page is in the handwriting of Philippe Dautzenberg (1849-1935), a Belgian amateur who lived in Paris and had a world-renowned collection, the contents of this handwritten catalogue of 55 pages is likely in Cousin's own hand. According to the summary included in the catalogue, his collection contained 16,141 shells, of which 10,007 were non-marine specimens from Ecuador. It gives not only a good impression of the extent of Cousin's collection, but also reveals how material listed in this catalogue ended up being described by Félix Pierre Jousseaume (1835-1921), whose collection is now in Paris (LAMY \& DAUTZENBERG 1952, VAN GEMERT 2007, VAN GEMERT \& BREURE 2017). An example is the species Mesembrinus vesperus Jousseaume, of which JOUSSEAUME (1887: 168) said "Un seul exemple de cette espèce à été receuilli par notre collège, M. A. Cousin, dans son propriété de los Puentès, près Quito" [A single specimen of this species was collected by our colleague A. Cousin, at his property Los Puentes near Quito]. As Jousseaume's paper was published in the same volume as Cousin's, and both mentioned in their introduction that Cousin's material had been the basis for each of them, it is safe to assume that any material in the former collection of Cousin may be regarded as type material for any taxon described by them; provided that it agrees with the data in their publications.

This paper documents the type material for species described by these two authors from the former collection of Cousin, which was sent to Jousseaume during Cousin's life time; on one of the labels he mentioned a letter of 14 September 1887 (Fig. 128). After Cousin's death his collection was bought in 1913 by Dautzenberg; a few shells were possibly traded via local Parisian shell dealers (likely the Deyrolle and Vimont companies). For completeness sake, the types described by Cousin and listed in BREURE (2011) are included in this survey. 


\section{METHODS}

Systematics follow MollusCABASE (2019) with families and taxa listed alphabetically. In the alphabetical treatment of taxa the original reference is given, published type locality, type material registrations and number of specimens, label information, remarks (if needed) and the current systematic posi-

\section{GENERAL REMARKS}

\section{BIOGRAPHICAL DATA}

Unfortunately there is but scant information about Auguste Édouard Cousin (Fig. 1; BREURE 2011: 3-5), which was partly based on an incorrect source. He was born 17 July 1835 in Paris (not Quito) and died 21 March 1899 in Quito (not Paris). He was the son of Edmé Paul Julien Cousin and Augustine Alexandrine Noël. He studied medicine in Paris and afterwards went to Ecuador, marrying Rosa Saá (1850-1928) in Quito in 1869; they had five children (COUSIN 2019). As may be clear from the introduction above, Cousin was friends with Jousseaume. The fact that the latter described an eponym for Cousin in 1877 is a sign that the contact between the two men was established already at that time. As the two men were of the same age, it is possible that they knew each other from school or study, but evidence is missing. Cousin became a member of the Société zoologique de France

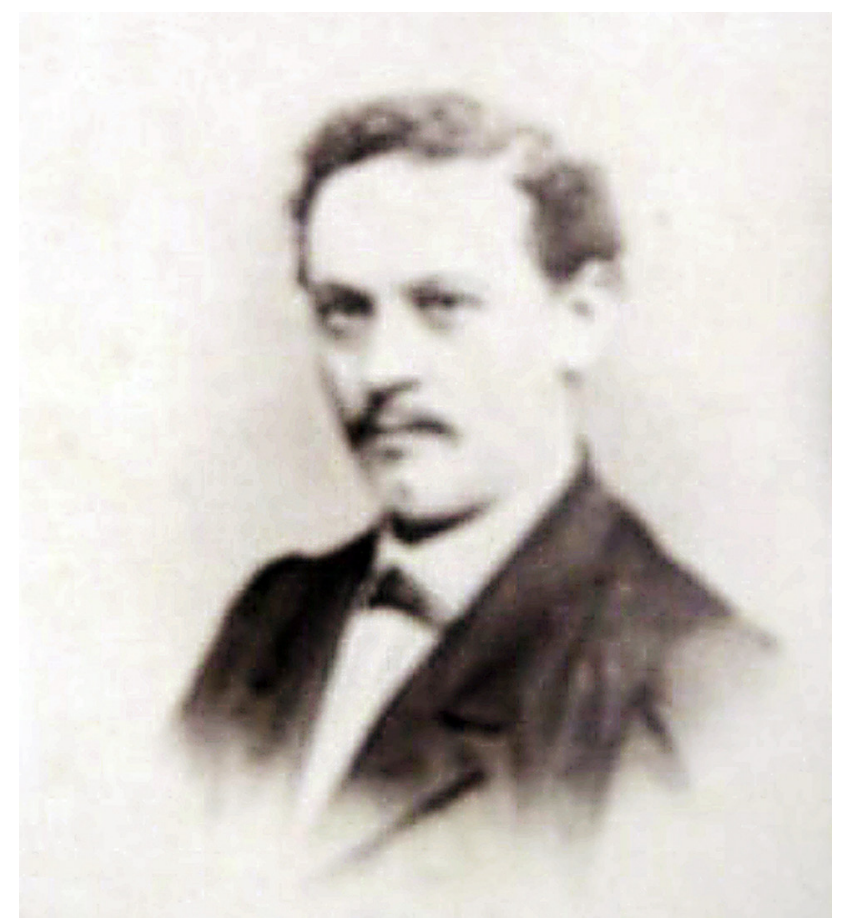

Fig. 1. Auguste Cousin at unknown age (modified after COUSIN 2019) tion as far as known. Figures of both the material and labels are provided. Abbreviations used for depositories: MNHN - Muséum national d'Histoire naturelle, Paris, France; RBINS - Royal Belgian Institute of Natural Science, Brussels, Belgium. Other abbreviations: $\mathrm{D}$ - shell diameter; $\mathrm{H}$ - shell height.

in 1882, with a Paris address (ANONYMOUS 1883). In 1889 he was mentioned as residing in Quito (ANONYMOUS 1889a) and as being awarded the medal 'Officier d'Académie' (ANONYMOUS 1889b: 350). Shortly afterwards his address was changed to Guayaquil (ANONYMOUS 1891: 10), until in 1897 he was listed in Quito again (ANONYMOUs 1897: 18) till his death in 1899 (ANONYMOUS 1900).

\section{MOLLUSCAN TAXA INTRODUCED BY A. E. COUSIN}

In his single molluscan publication he described the following taxa as new to science, which are partly treated in more detail below (marked with an asterisk*).

*Ammonoceras cyclina COUSIN 1887: 245, pl. 4 fig. 6.

*Ampullaria puntaplaya CoUsin 1887: 278, pl. 4 fig. 2.

*Ampullaria reyrei COUSIN 1887: 279, pl. 4 fig. 7.

Aplecta gualbertoi COUSIN 1887: 261, pl. 4 fig. 3.

${ }^{*}$ Cyclophorus antonii COUSIN 1887: 271, pl. 4 fig. 4.

*Hamadryas fallax var. major COUSIN 1887: 219.

*Isomeria bourcieri var. lutea COUSIN 1887: 255.

${ }^{*}$ Neritostoma marianita COUSIN 1887: 236, pl. 4 figs 5, 5 bis.

Obeliscus cuneus var. minor COUSIN 1887: 237 [nomen nudum].

Obeliscus cuneus var. major COUSIN 1887: 237 [nomen nudum].

Obeliscus jousseaumi COUSIN 1887: 238, pl. 4 fig. 1.

*Orphnus thompsoni var. lutea COUSIN 1887: 212.

*Orphnus thompsoni var. nigricans COUSIN 1887: 212.

*Orphnus thompsoni var. olivacea COUSIN 1887: 212.

*Orphnus thompsoni var. zebra COUSIN 1887: 212.

*Planorbis canonicus COUSIN 1887: 264, pl. 4 fig. 11.

*Planorbis equatorius COUSIN 1887: 263, pl. 4 fig. 8.

Porphyrobaphe irrorata var. $\beta$ elongata COUSIN 1887: 203 [nomen nudum].

Thaumastus aequatorius var. fasciata COUSIN 1887: 222 [nomen nudum].

Thaumastus aequatorius var. immaculata COUSIN 1887: 222 [nomen nudum].

Thaumastus aequatorius var. interrupta COUSIN 1887: 222 [nomen nudum]. 
Thaumastus aequatorius var. major COUSIN 1887: 222 [nomen nudum].

*Thaumatus alausiensis COUSIN 1887: 228, pl. 4 fig. 13. * Thaumatus juana COUSIN 1887: 228, pl. 4 fig. 10.

*Thaumastus (?) nystianus var. lutea COUSIN 1887: 220.

*Thaumastus (?) nystianus var. nigricans COUSIN 1887: 221.

Veronicella marianita COUSIN 1887: 190, pl. 4 fig. 14.

\section{EPONYMS NAMED AFTER A. E. COUSIN}

The following eponyms were given to Auguste Cousin (this list is not necessarily complete):

Ampullaria cousini JOUSSEAUME 1887: 185, pl. 3 fig. 3.

[Gastropoda, Ampulariidae].

\section{SYSTEMATIC PART}

\section{ALPHABETICAL LIST OF FAMILIES WITH COUSIN'S AND JOUSSEAUME'S NEW TAXA}

\section{Family Achatinidae Swainson, 1840} jousseaumei Cousin, 1887 (Obeliscus); valenzuela Jousseaume, 1887 (Leptinaria).

\section{Family Ampullariidae J. E. Gray, 1824}

puntaplaya Cousin, 1887 (Ampullaria); reyrei Cousin, 1887 (Ampullaria); tenuissima Jousseaume, 1894 (Ampullaria).

\section{Family Bulimulidae Tryon, 1867}

alausiensis Cousin (Thaumastus), 1887; juana Cousin, 1887 (Thaumastus); major Cousin, 1887 (Hamadryas); nigricans Cousin, 1887 (Thaumastus).

\section{Family Labyrinthidae Borrero, Sei, Robinson et Rosenberg, 2017 lutea Cousin, 1887 (Isomeria).}

\section{Family Lymnaeidae Rafinesque, 1815} cousini Jousseaume, 1887 (Limnaea); raphaelis Jousseaume, 1887 (Limnaea).

\section{Family Neocyclotidae Kobelt et Möllendorff,} 1897

antonii Cousin, 1887 (Cyclophorus); vasconesi Jousseaume, 1897 (Cyclophorus).

Family Orthalicidae Martens in Albers, 1860 lutea Cousin, 1887 (Orphnus); nigricans Cousin, 1887 (Orphnus); olivacea Cousin, 1887 (Orphnus); zebra Cousin, 1887 (Orphnus).

\section{Family Planorbidae Rafinesque, 1815}

canonicus Cousin, 1887 (Planorbis); clevei Jousseaume in Cousin, 1887 (Planorbis); equatorius Cousin, 1887 (Planorbis).
Biomphalaria cousini PARAENSE 1966: 119, figs 2, 4, 6. [Gastropoda, Planorbidae].

Cyclophorus cousini JOUSSEAUME 1884: 173, pl. 4 fig. 13. [Gastropoda, Neocyclotidae].

Lymnaea cousini JOUSSEAUME 1887: 182, pl. 3 fig. 8. [Gastropoda, Lymnaeidae].

Nenia cousini JOUSSEAUME 1900: 32, pl. 1 fig. 12. [Gastropoda, Clausiliidae].

Proserpinella cousini JOUSSEAUME 1887: 181, pl. 13 figs 15-16. [Gastropoda, Proserpinellidae].

Rhodea cousini JOUSSEAUME 1900: 36, pl. 1 fig. 15.

[Gastropoda, Achatinidae].

Solaropsis cousini JOUSSEAUME 1887: 177. [Gastropoda, Solaropsidae].

Family Proserpinellidae H. B. Baker, 1923 cousini Jousseaume, 1887 (Proserpinella).

Family Scolodontidae H. B. Baker, 1925 locardi Jousseaume, 1887 (Guestieria); lyzarzaburui Jousseaume, 1887 (Ammonoceras); martinida Jousseaume, 1887 (Guestieria).

Family Solaropsidae H. Nordsieck, 1986 cousini Jousseaume, 1887 (Solaropsis); cyclina Cousin, 1887 (Ammonoceras).

Family Succineidae H. Beck, 1837

marianita Cousin, 1887 (Neritostoma).

\section{ALPHABETICAL LIST OF TAXA}

\section{alausiensis, Thaumastus Cousin, 1887 [Bulimulidae]}

Figs 2-6

Thaumastus alausiensis COUSIN 1887: 228, pl. 4 fig. 13.

Type locality. "sur le versant du mont Hacu, entre Achapallas et la rivière Sula, sur le territoire Alausi, province de Chimborazo" [on the slope of Mount Hacu, between Achapallas and the River Sula, in the Alausi territory, province of Chimborazo].

Dimensions. "long. 25mm; diam. 9 à $10 \mathrm{~mm}$ "; figured specimen $\mathrm{H} 25.7 \mathrm{~mm}$.

Type material. RBINS MT.2333 (1), MT.2334 (12), paralectotypes.

Label. "Racu, descente de Achupulla au Rio Sula" [Racu, descent from Achupulla to Rio Sula].

Type evidence. The material is ex Dautzenberg ex Cousin collection, label in Cousin's handwriting.

Remarks. Additional type material is in the Paris museum: MNHN-IM-2000-20820 (lectotype; BREURE 1976: 1141); MNHN-IM-2000-20821 (1), paralectotype. 

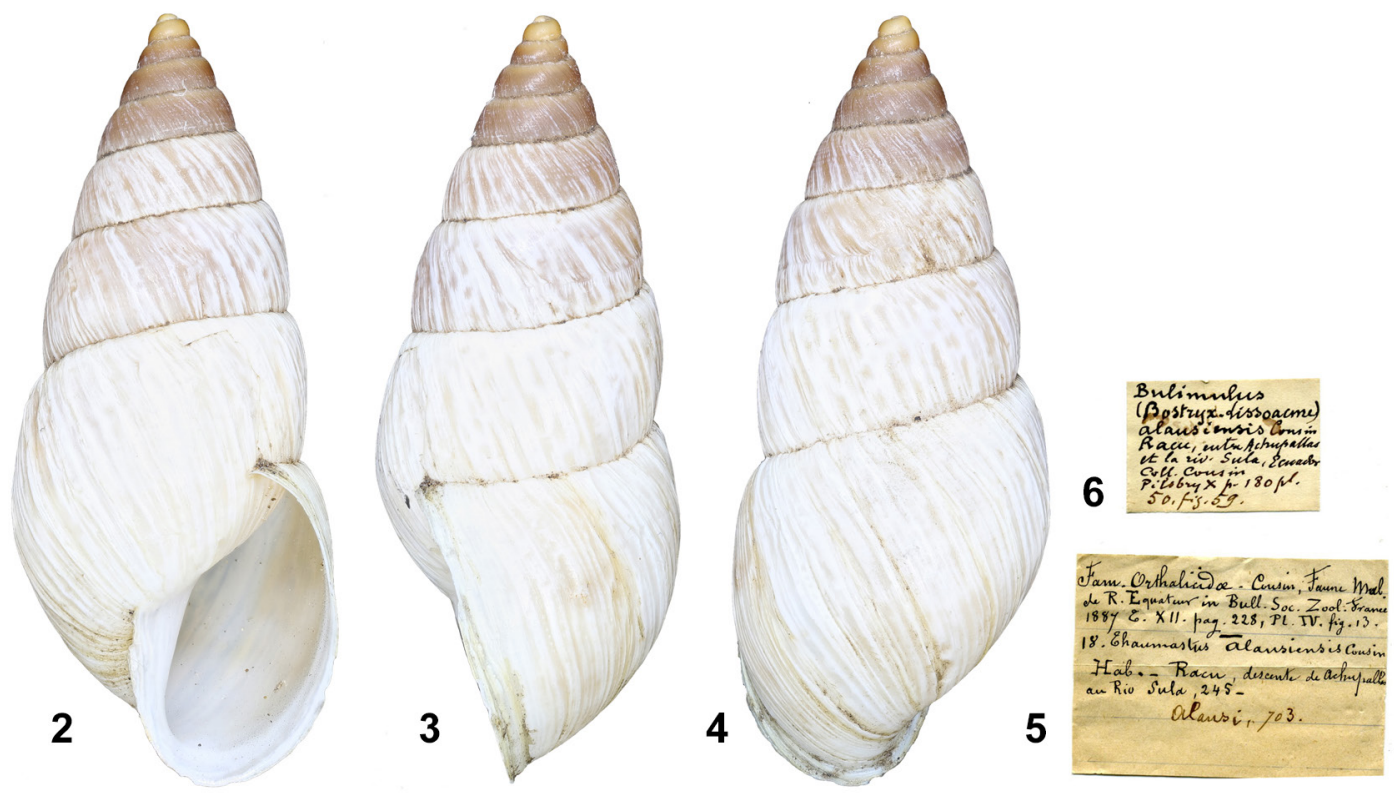

Figs 2-6. Bostryx alausiensis (Cousin, 1887), paralectotype RBINS MT.2333 (H 25.7 mm): 2 - apertural view, 3 - right lateral view, 4 - dorsal view, 5 - original label by Cousin, 6 - label by Dautzenberg
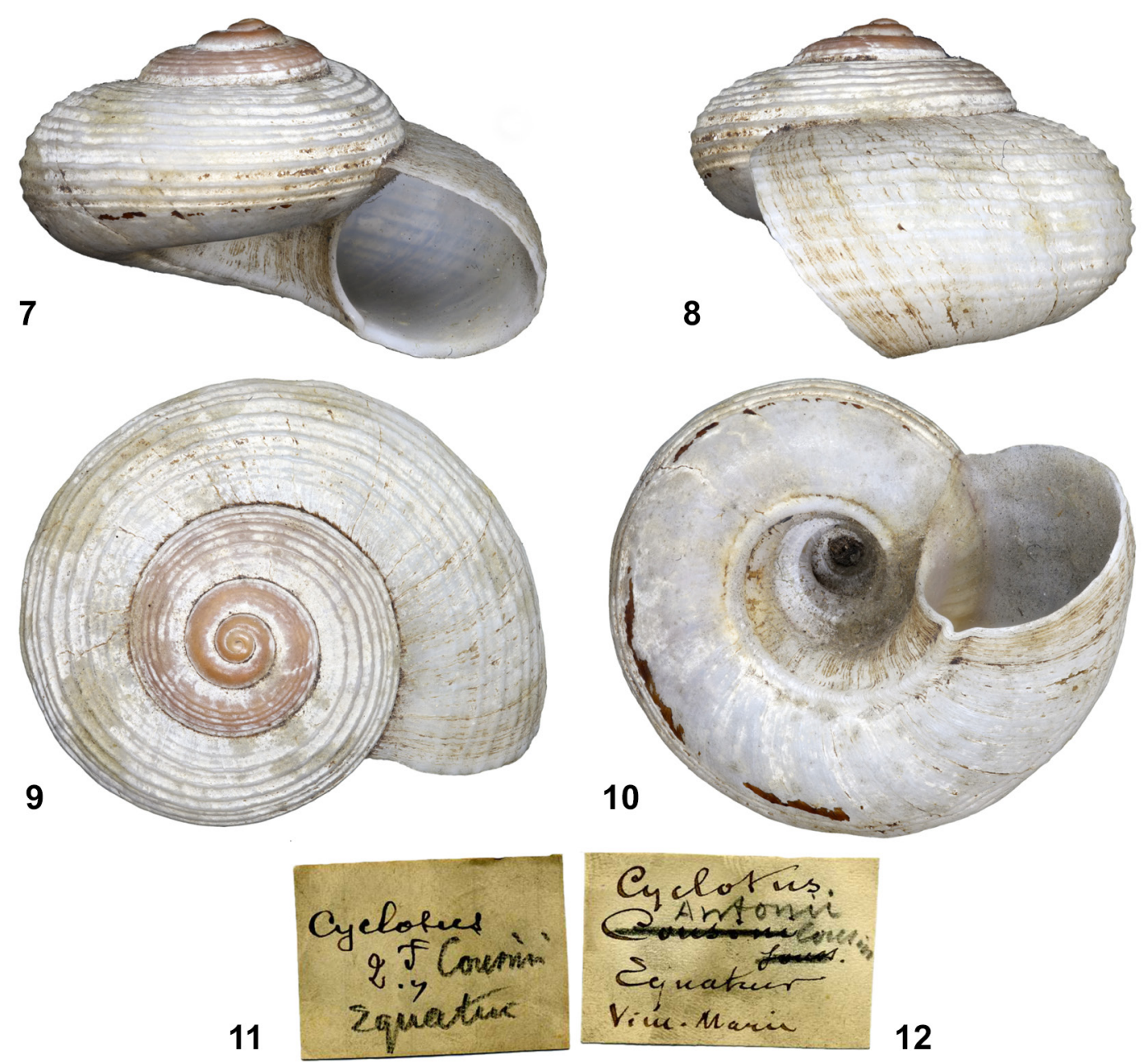

Figs 7-12. Lagocyclus antonii (Cousin, 1887), syntype RBINS MT.3816 (D $21.7 \mathrm{~mm}$ ): 7 - apertural view, 8 - right lateral view, 9 - apical view, 10 - umbilical view, 11 - label by Dautzenberg, 12 - same 
Current systematic position. Bostryx alausiensis (Cousin, 1887).

\section{antonii, Cyclophorus Cousin, 1887 [Neocyclotidae]}

Figs 7-12

Cyclophorus antonii COUSIN 1887: 271, pl. 4 fig. 4.

Type locality. "je connais pas l'habitat exact" [I don't know the exact habitat].

Dimensions. "gr. diam., $20-21^{\mathrm{mm}}$; p. diam., $17-18^{\mathrm{mm}}$;

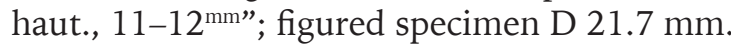
Type material. RBINS MT.3816 (2), syntypes. Label. "Équateur".

Type evidence. The material is ex Dautzenberg ex Cousin collection, label in Dautzenberg's handwriting. The original label is lost. The taxon name on the current label was originally a Jousseaume name [Cyclotus cousini $=$ Calaperostoma cousini (Jousseaume, 1884)], also in Dautzenberg's hand, which has been corrected by him. The lowest row on the label might refer to a locality, but could not be found with modern gazetteers. The alternative is a reference to a provenance, in which case it might refer to the dealer Vimont where E. Marie worked in his later years.

Current systematic position. Lagocyclus antonii (Cousin, 1887).

\section{canonicus, Planorbis Cousin, 1887 [Planorbidae]}

Figs 13-16

Planorbis canonicus COUSIN 1887: 264, pl. 4 fig. 11.

Type locality. "le lac Saint-Paolo, près Quito" [Lake St. Paolo, near Quito].

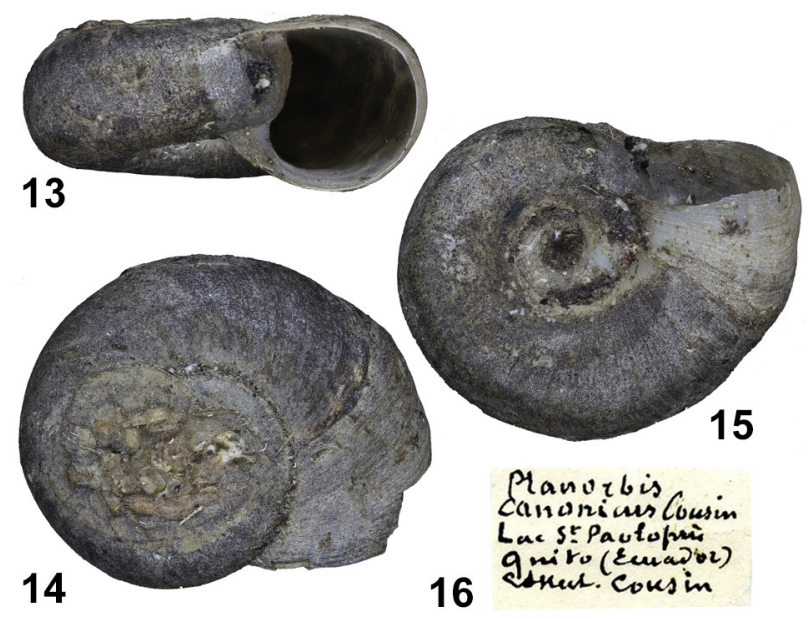

Figs 13-16. Biomphalaria peregrina (d'Orbigny, 1835), syntype of Planorbis canonicus Cousin, 1887 RBINS MT.3852 (D $9.8 \mathrm{~mm}$ ): 13 - apertural view, 14 - apical view, 15 umbilical view, 16 - label by Dautzenberg
Dimensions. "gr. diam., $10^{\mathrm{mm}}$; pet. diam., $8^{\mathrm{mm}}$; épaisseur, $4^{\mathrm{mm}}$; figured specimen D $9.8 \mathrm{~mm}$.

Type material. RBINS MT.3852 (20), syntypes.

Label. "Lac St Paulo près Quito (Ecuador)", "Lac San Pablo, Ecuador".

Type evidence. The material is ex Dautzenberg ex Cousin collection, the labels are both in the handwriting of Dautzenberg.

Remarks. The taxonomic position has been revised by PARAENSE (2004).

Current systematic position. Biomphalaria peregrina (d'Orbigny, 1835) (PARAENSE 2004).

clevei, Planorbis Jousseaume in Cousin, 1887 [Planorbidae]

Figs 17-22

Planorbis clevei Jousseaume in COUSIN 1887: 263, pl. 4 fig. 9.

Type locality. "Équateur".

Dimensions. "gr. diam., $18^{\mathrm{mm}}$; pet. diam., $15^{\mathrm{mm}}$; épaisseur, $6^{\mathrm{mm}} 5$ "; figured specimen D $18.2 \mathrm{~mm}$.

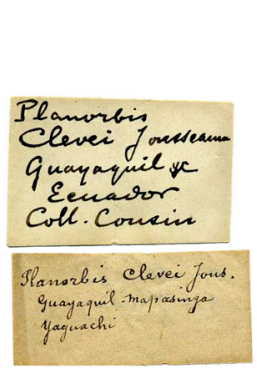

20
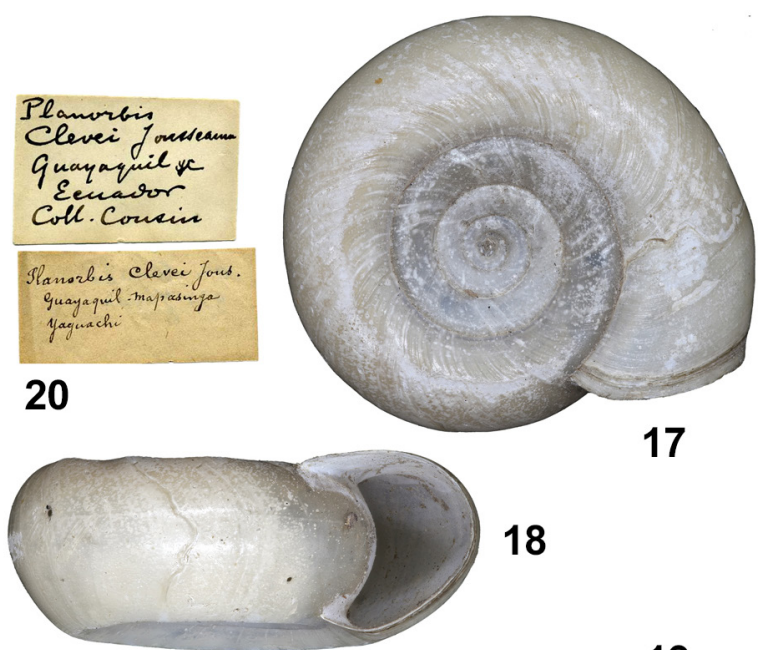

18

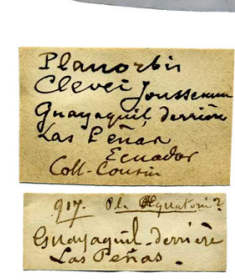

21

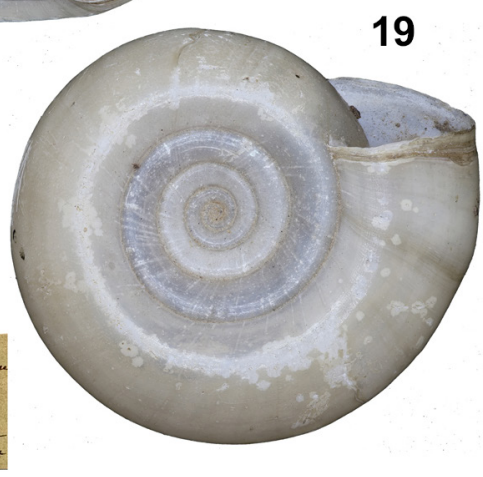

22

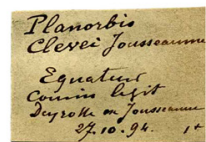

Figs 17-22. Biomphalaria clevei (Jousseaume, 1887), syntype RBINS MT.3841 (D $18.2 \mathrm{~mm}$ ): 17 - apical view, 18 - apertural view, 19 - umbilical view, 20 - labels Dautzenberg respectively Jousseaume, 21 - labels Dautzenberg respectively Cousin, 22 - label by Dautzenberg 
Type material. RBINS MT.3841 (1), MT.3842 (3), MT.3843 (7), MT.3844 (9), syntypes.

Label. "Équateur", "Guayaquil, Mapasinga, Yaguachi", "Guayaquil, derrière Las Peñas" "Boca de Estero de Zarumilla, province de Oro".

Type evidence. The material is ex Dautzenberg ex Cousin collection; two labels are written presumably in Jousseaume's hand, three later labels written in Dautzenberg's handwriting (one label mentioning "Cousin leg. / Deyrolle ex Jousseaume").

Remarks. The specimen which is ex Jousseaume is much smaller $(13.1 \mathrm{~mm})$ than the original dimensions ("gr. diam. $18 \mathrm{~mm}$ "). This taxon has not been revised by later authors.

Current systematic position. Unknown, provisionally Biomphalaria clevei (Jousseaume, 1887).

\section{cousini, Limnaea Jousseaume, 1887 [Lymnaeidae]}

Figs 23-27

Limnaea cousini JOUSSEAUME 1887: 182, pl. 3 fig. 8.

Type locality. "Chancu-Yacu, près de Chillogallo, canton de Quito" [Chancu-Yacu, near Chillogallo, canton of Quito].

Dimensions. "long., 10 à $14^{\mathrm{mm}}$; gr. diam., 6 à $10^{\mathrm{mm}}$; p. diam. 5 à $6^{\mathrm{mm}}$; ouverture, long., 7 à $10^{\mathrm{mm}}$; larg., 4 à $6^{\mathrm{mm}}$; figured specimen $\mathrm{H} 9.1 \mathrm{~mm}$.

Type material. RBINS MT.3847 (20), syntypes.

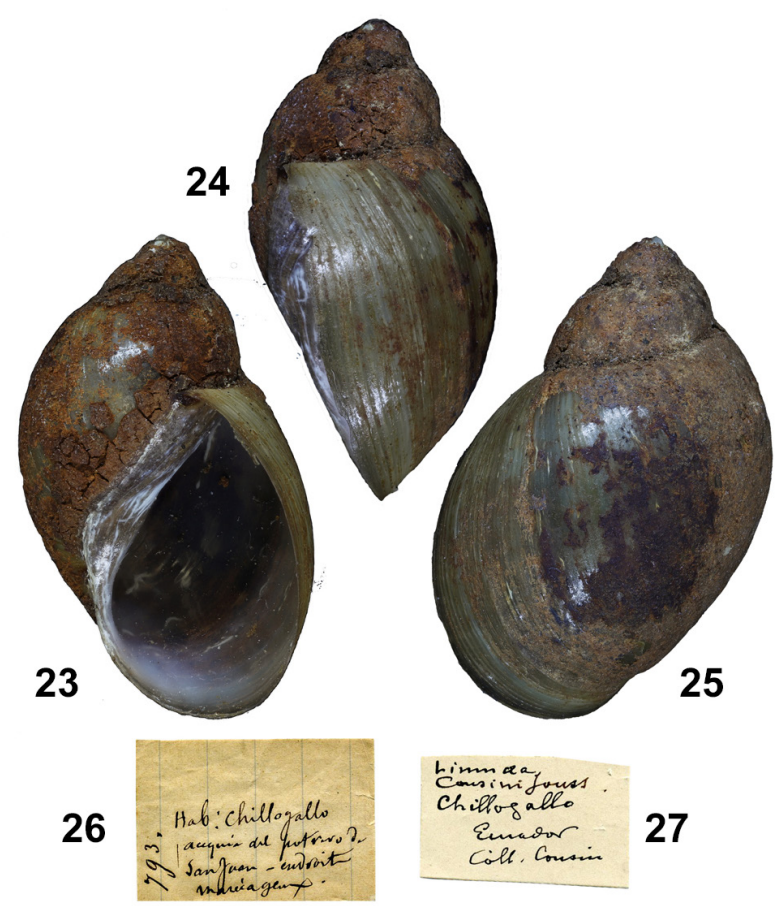

Figs 23-27. Galba cousini (Jousseaume, 1887), syntype RBINS MT.3847 (H $9.1 \mathrm{~mm}$ ): 23 - apertural view, 24 - right lateral view, 25 - dorsal view, 26 - label presumably by Cousin, 27 - label by Dautzenberg
Label. "Chillogallo".

Type evidence. The material is ex Dautzenberg ex Cousin collection; label presumably in Cousin's handwriting.

Remarks. Additional type material is present in the Paris museum: MNHN-IM-2000-35288 (8), MNHNIM-2000-35289 (12). According to PARAENSE (2004) this is a valid species.

Current systematic position. Galba cousini (Jousseaume, 1887).

\section{cousini, Proserpinella Jousseaume, 1887 [Proserpinellidae]}

Figs 28-33

Proserpinella cousini JOUSSEAUME 1887: 181, pl. 3 figs 15-16.

Type locality. "République de l'Équateur".

Dimensions. "gr. diam., $13^{\mathrm{mm}}$; p. diam., $11^{\mathrm{mm}}$; haut., $7^{\mathrm{mm}}$; figured specimen D $10.0 \mathrm{~mm}$.

Type material. RBINS MT.43 (3), syntypes.

Label. "Los Puentes San Fernandino".

Type evidence. The material is ex Dautzenberg ex Cousin collection; two labels written in Cousin's handwriting.

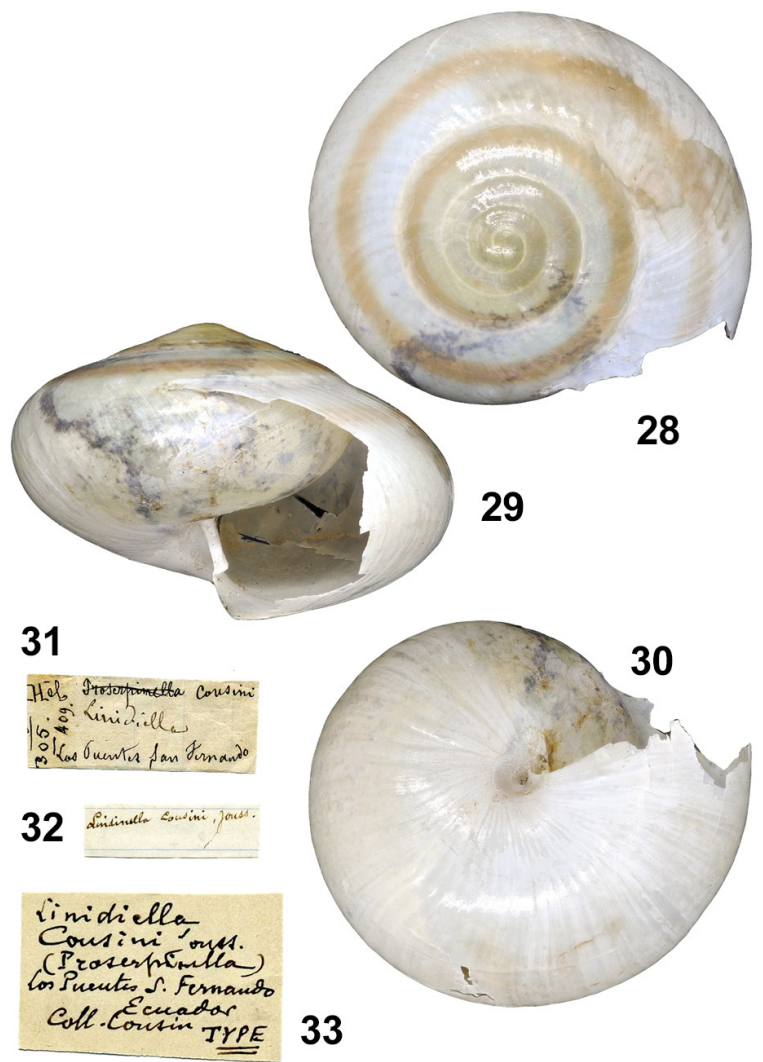

Figs 28-33. Archecharax cousini (Jousseaume, 1887), syntype RBINS MT.43 (D $10.0 \mathrm{~mm}$ ): 28 - apical view, 29 - apertural view, 30 - umbilical view, 31 - label by Jousseaume, 32 - same, 33 - label by Dautzenberg 
Remarks. JOUSSEAUME (1887: 182) remarked "Je ne puis pas indiquer le point précis de la République de l'Équateur où cette espèce a été trouvée" [I cannot indicate the precise place within Ecuador where this species has been found]. The label text seems to refer to two localities: 'Los Puentes', a locality where Cousin lived, and 'San Fernandino', which could not be found with modern gazetteers. The aperture is damaged, which explains the differences in dimensions between the published and the actual data.

Current systematic position. Archecharax cousini (Jousseaume, 1887).

\section{cousini, Solaropsis Jousseaume, 1887 [Solaropsidae]}

\section{Figs 34-37}

Solaropsis cousini JOUSSEAUME 1887: 177.

Type locality. "le bassin du Napo, Équateur" [Napo Basin, Ecuador].

Dimensions. "gr. diam., $85^{\mathrm{mm}}$; p. diam., $70^{\mathrm{mm}}$; haut. $45^{\mathrm{mm}}$; figured specimen D $84.6 \mathrm{~mm}$.

Type material. RBINS MT.3851 (4), syntypes.

Label. "Napo" "Ávila, 440' / Toplat [?] de Napo, 264'

/ Tena, 511”".
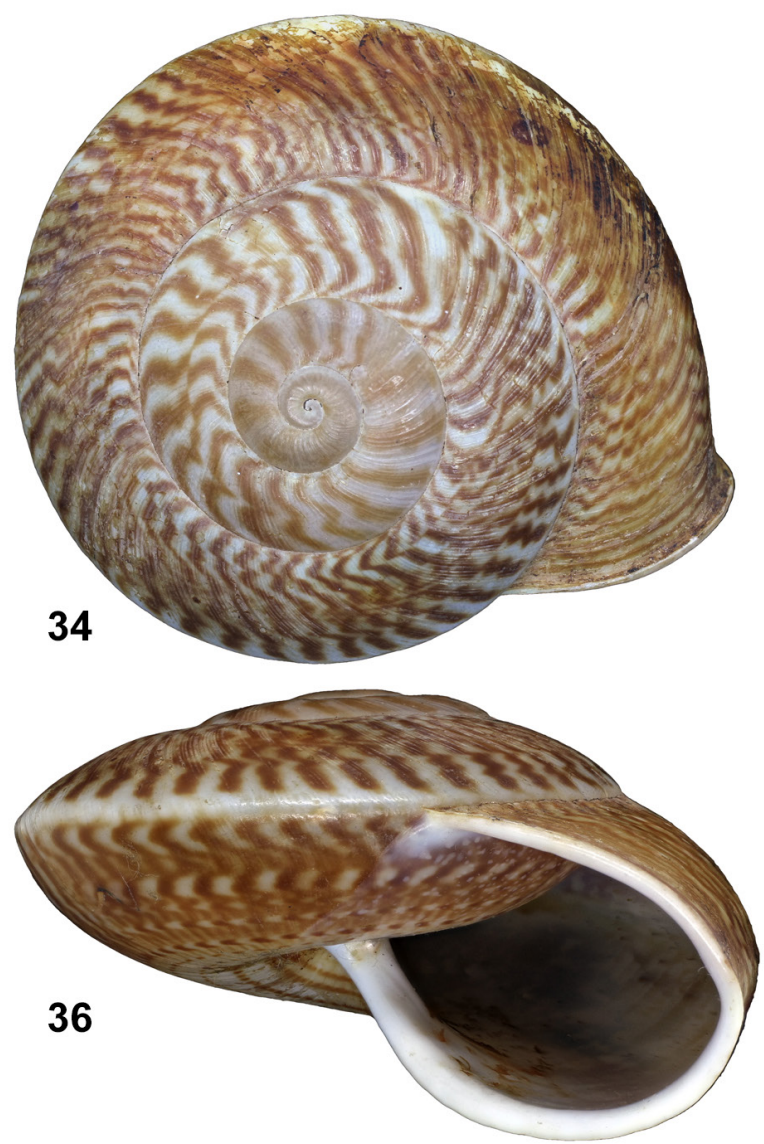

Type evidence. The material is ex Dautzenberg ex Cousin collection; label written in Cousin's handwriting.

Remarks. As far as I know, this species has not been figured before. It is very similar to Solaropsis gibboni (L. Pfeiffer, 1846), described from 'Neu-Grenada', and further research may show it to be a junior subjective synonym of Pfeiffer's taxon.

Current systematic position. Solaropsis cousini Jousseaume, 1887.

\section{cyclina, Ammonoceras Cousin, 1887 [Systrophiidae]}

Figs 38-42

Ammonoceras cyclina Cousin 1887: 245, pl. 4 fig. 6.

Type locality. Not given.

Dimensions. "gr. diam., 20 à $21^{\mathrm{mm}}$; pet. diam., 18 à $19^{\mathrm{mm}}$; haut., 6 à $7^{\mathrm{mm}}$; ouverture, 6 à $7^{\mathrm{mm}}$; figured specimen D $17.2 \mathrm{~mm}$.

Type material. RBINS MT.3839 (2), syntypes.

Label. "Hac. [Hacienda] Puca Yacu près Quevedo" [Puca Yacu farm near Quevedo].

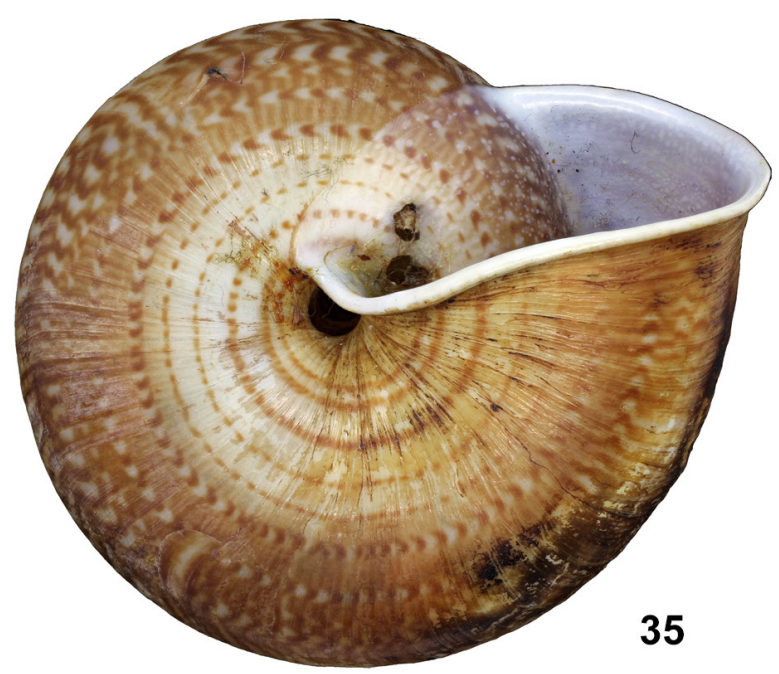

Figs 34-37. Solaropsis cousini Jousseaume, 1887, syntype RBINS MT.3851 (D 84.6 mm): 34 - apical view, 35 - umbilical view, 36 - apertural view, 37 - label by Jousseaume 


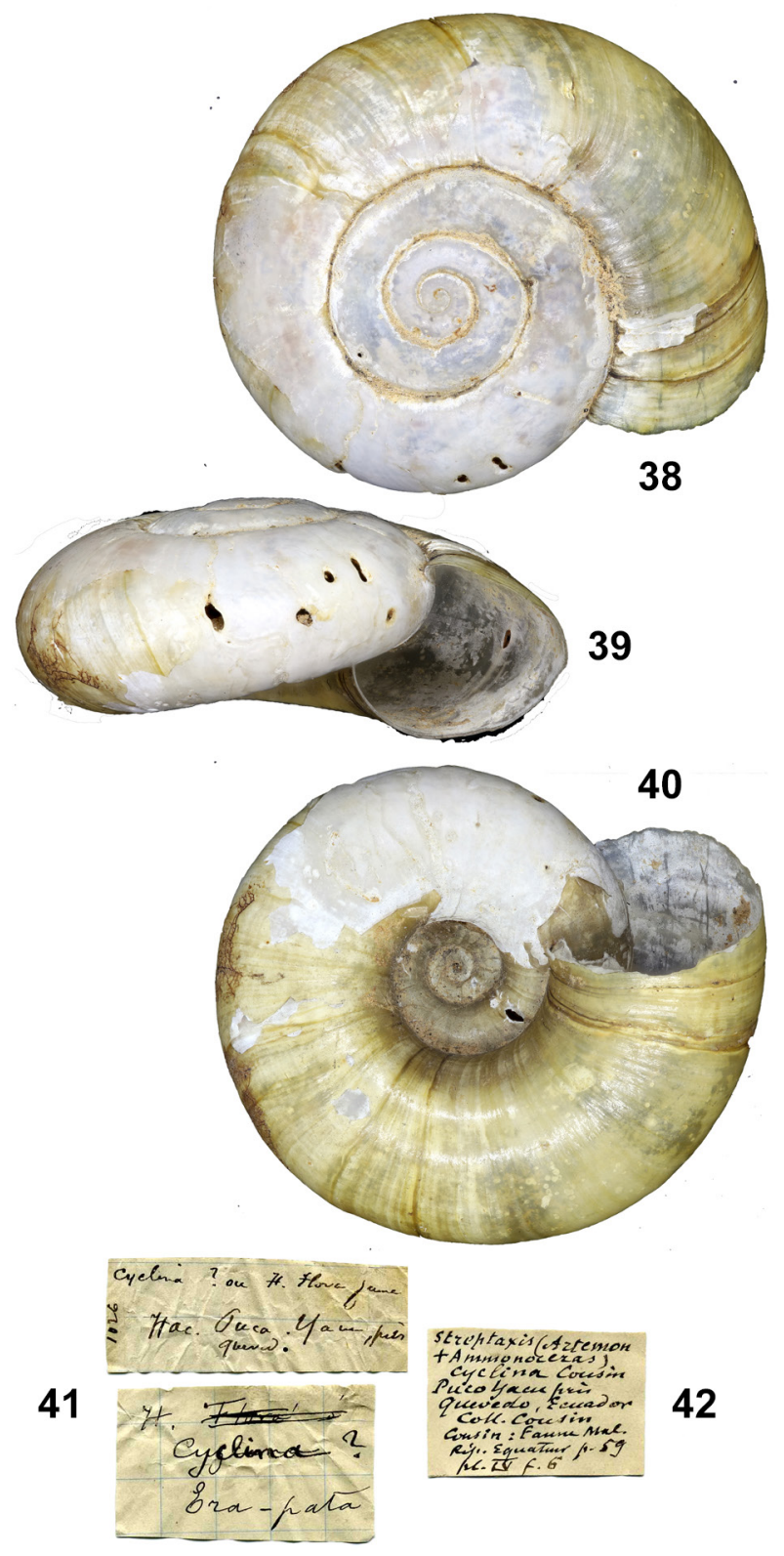

Figs 38-42. Happia cyclina (Cousin, 1887), syntype RBINS MT.3839 (D $17.2 \mathrm{~mm}$ ): 38 - apical view, 39 - apertural view, 40 - umbilical view, 41 - labels by Cousin, $42-$ label by Dautzenberg
Type evidence. The material is ex Dautzenberg ex Cousin collection; label presumably written in Jousseaume's handwriting.

Current systematic position. Happia cyclina (Cousin, 1887).

\section{equatorius, Planorbis Cousin, 1887 [Planorbidae]}

Figs 43-48

Planorbis equatorius COUSIN 1887: 263, pl. 4 fig. 8.

Type locality. "Équateur".

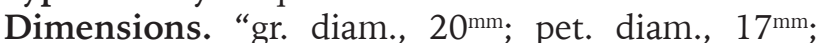
épaisseur, 6 ${ }^{\mathrm{mm}}$ 5"; figured specimen D $22.3 \mathrm{~mm}$.

Type material. RBINS MT.3831 (10), MT.3832 (10), MT.3833 (5), MT.3834 (14), syntypes.

Labels. "environs de Guayaquil", "Milagro", "Guayaquil, [illigible]", "Yaguachi".

Type evidence. All material originates from the Cousin collection, partly with original labels.

Remarks. The specimen now figured bears a strong resemblance to the original figure by Cousin. PARAENSE (2004) considered this taxon as a valid species.

Current systematic position. Biomphalaria equatoria (Cousin, 1887).

\section{jousseaumei, Obeliscus Cousin, 1887 [Achatinidae]}

Figs 49-52

Obeliscus jousseaumei COUSIN 1887: 238, pl. 4 fig. 1.

Type locality. "à la descente d'Azagues, venant de Molobog (...) à une altitude d'environs 2,400 mèt." [on the descent of Azagues, coming from Molobog (...) at an altitude of about 2,400 meters].

Dimensions. "long., $27^{\mathrm{mm}}$; diam., $8^{\mathrm{mm}}$; ouverture: long., $8^{\mathrm{mm}}$; larg., $5^{\mathrm{mm}}$; figured specimen H $28.3 \mathrm{~mm}$.

Type material. RBINS MT.3845 (28), syntypes.

Label. "Foret Azogues No 177 - Azogues, Cuenca, 192 - Chemin à Gualea 322 - Chicticay 149 Gualaceo 229".
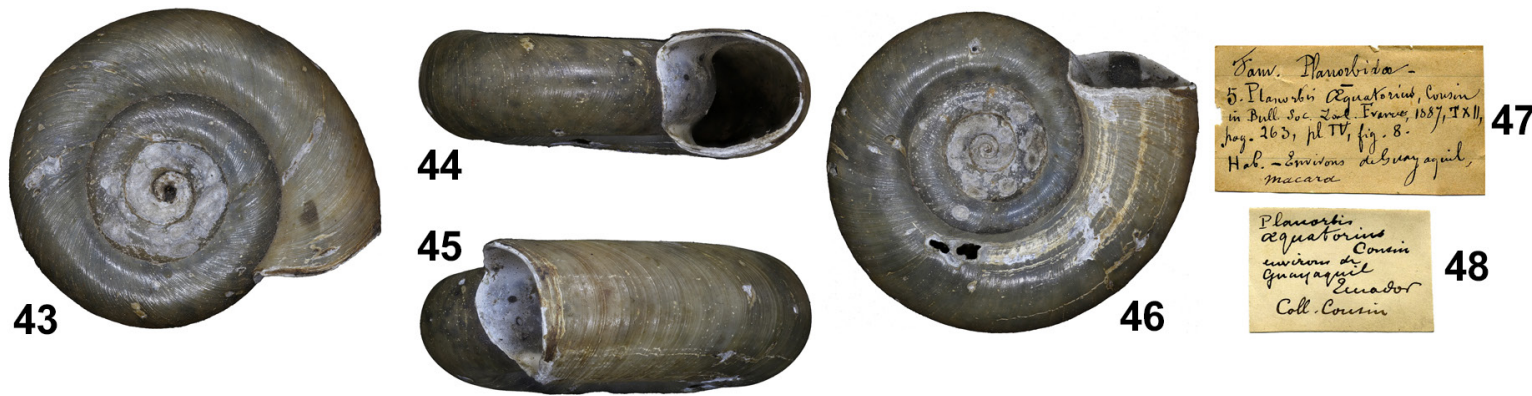

Figs 43-48. Biomphalaria equatoria (Cousin, 1887), syntype RBINS MT.3831 (D 22.3 mm): 43 - apical view, 44 - apertural view, 45 - right lateral view, 46 - umbilical view, 47 - label by Cousin, 48 - label by Dautzenberg 


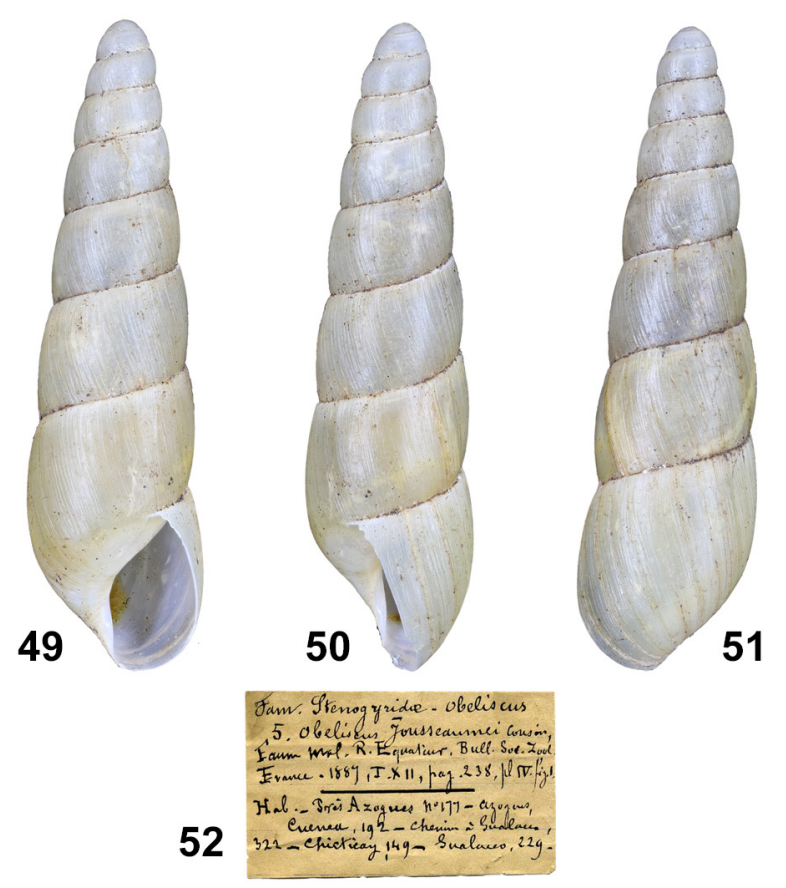

Figs 49-52. Protobeliscus jousseaumi (Cousin, 1887), syntype RBINS MT.3845 (H 28.3 mm): 49 - apertural view, 50 - right lateral view, 51 - dorsal view, 52 - label by Dautzenberg

Type evidence. The material is ex Dautzenberg ex Cousin collection; label written in Cousin's handwriting.

Remarks. The label suggests that this lot is a mixture from different localities, however, they cannot be disentangled anymore.

Current systematic position. Protobeliscus jousseaumi (Cousin, 1887).

\section{juana, Thaumastus Cousin, 1887 [Bulimulidae]}

Figs 53-57

Thaumastus juana COUSIN 1887: 228, pl. 4 fig. 10.

Type locality. "Gualacco, province de Cuença". Dimensions. "long., 20 à $23^{\mathrm{mm}}$; diam. 10 à $12^{\mathrm{mm}}$; figured specimen $\mathrm{H} 19.1 \mathrm{~mm}$.

Type material. RBINS MT.2357 (7), paralectotypes. Label. "chemin à Gualacco, rives du Paute, avant le pont" [path to Gualacco, banks of the Paute, before the bridge].

Type evidence. The material is ex Dautzenberg ex Cousin collection; label in Cousin's handwriting. Remarks. Additional type material is in the Paris museum: MNHN-IM-2000-21318 (lectotype; BREURE 1976: 1141).

Current systematic position. Bostryx juana (Cousin, 1887).

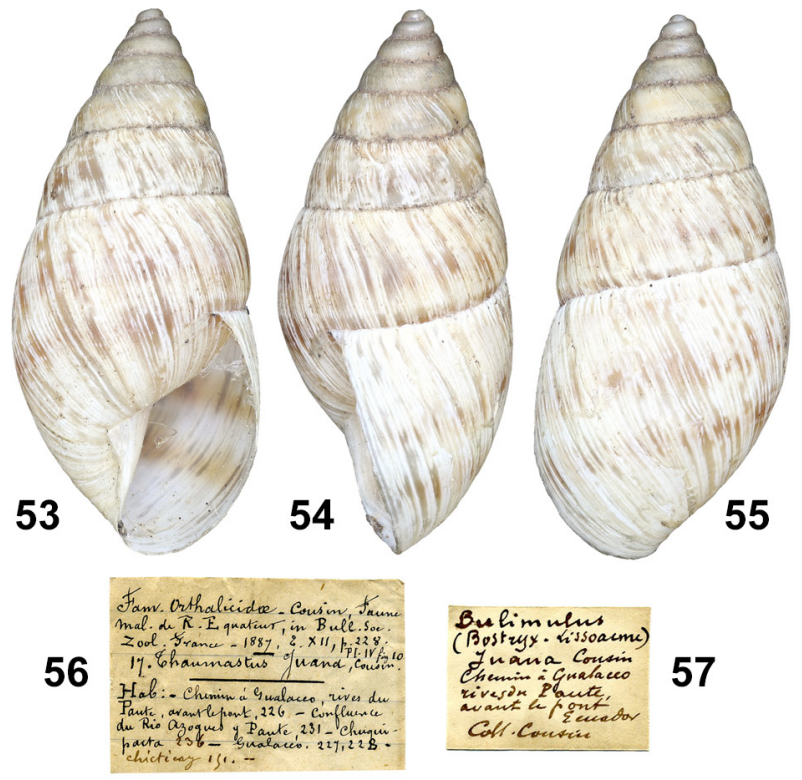

Figs 53-57. Bostryx juana (Cousin, 1887), paralectotype RBINS MT.2357 (H $19.1 \mathrm{~mm}$ ): 53 - apertural view, 54 right lateral view, 55 - dorsal view, 56 - label by Cousin, 57 - label by Dautzenberg

\section{locardi, Guestieria Jousseaume, 1887 [Scolodontidae]}

Figs 58-62

Guestieria locardi JOUSSEAUME 1887: 175, pl. 3 fig. 13.

Type locality. "Los Puantès [sic], canton de Quito". Dimensions. "gr. diam., $9^{\mathrm{mm}}$; p. diam., $7^{\mathrm{mm}}$; haut., $4^{\mathrm{mm}}$; figured specimen D $8.4 \mathrm{~mm}$.

Type material. RBINS MT.3835 (3), syntypes.

Label. "Los Puentes".

Type evidence. The material is ex Dautzenberg ex Cousin collection; label written in Dautzenberg's handwriting.

Remarks. Additional type material is in the Paris museum: MNHN-IM-2000-25757 (9), syntypes.

Current systematic position. Guestieria locardi Jousseaume, 1887.

\section{lutea, Isomeria bourcieri Cousin, 1887 [Labyrinthidae]}

\section{Figs 63-68}

Isomeria bourcieri var. lutea COUSIN 1887: 255.

Type locality. "Los Puentes près de Gualea".

Dimensions. Not given; figured specimen D $31.8 \mathrm{~mm}$.

Type material. RBINS MT.3817 (9), syntypes.

Label. "Ecuador, Los Puentes".

Type evidence. The material is ex Dautzenberg ex Cousin collection; label written in Dautzenberg's handwriting, locality label in Cousin's hand.

Remarks. This is a colour form of the nominate species. 


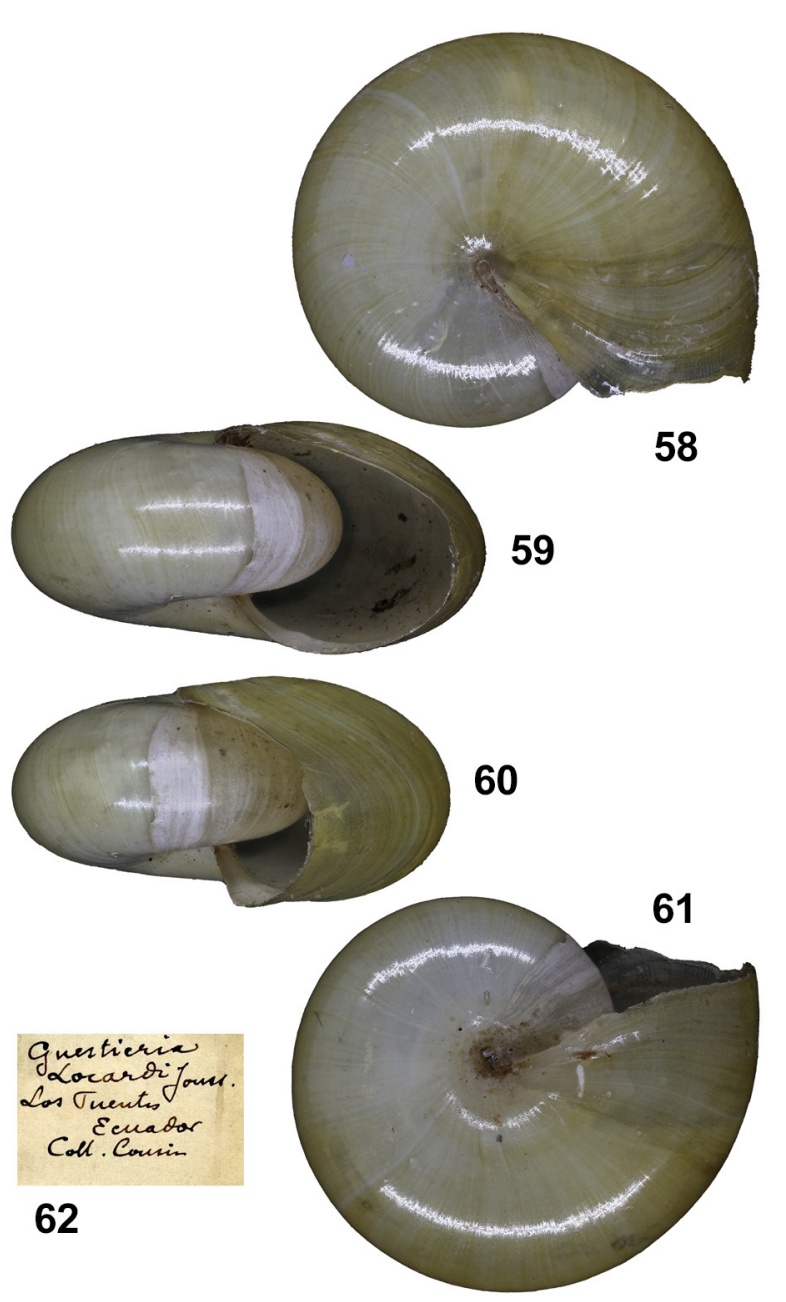

Figs 58-62. Guestieria locardi Jousseaume, 1887, syntype RBINS MT.3835 (D 8.4 mm): 58 - apical view, 59 - apertural view, 60 - right lateral view, 61 - umbilical view, 62 - label by Dautzenberg
Current systematic position. Isomeria bourcieri (L. Pfeiffer, 1853) (syn. n.).

\section{lutea, Orphnus thompsoni Cousin, 1887 [Orthalicidae]}

Figs 69-72

Orphnus thompsoni var. lutea COUSIN 1887: 212.

Type locality. "Cuença".

Dimensions. Not given; figured specimen $\mathrm{H}$ $75.6 \mathrm{~mm}$.

Type material. RBINS MT.2358 (lectotype; BREURE 2011: 32); RBINS MT.2359 (5), paralectotypes.

Label. "Cuenca Ecuador".

Type evidence. The material is ex Dautzenberg ex Cousin collection; label written in Dautzenberg's handwriting.

Current systematic position. Kara thompsonii (L. Pfeiffer, 1845) (BREURE 2011).

\section{lutea, Thaumastus (?) nystianus Cousin, 1887 [Bulimulidae]}

Figs 73-74

Thaumastus (?) nystianus var. lutea COUSIN 1887: 220.

Type locality. "près Quito (...) Tumbaco (...) CashaLoma, près Chillogallo" [see remarks].

Dimensions. Not given; figured specimen $\mathrm{H}$ $36.1 \mathrm{~mm}$.

Type material. RBINS MT.3818 (3), possible syntypes. Label. "Chilguiltina".
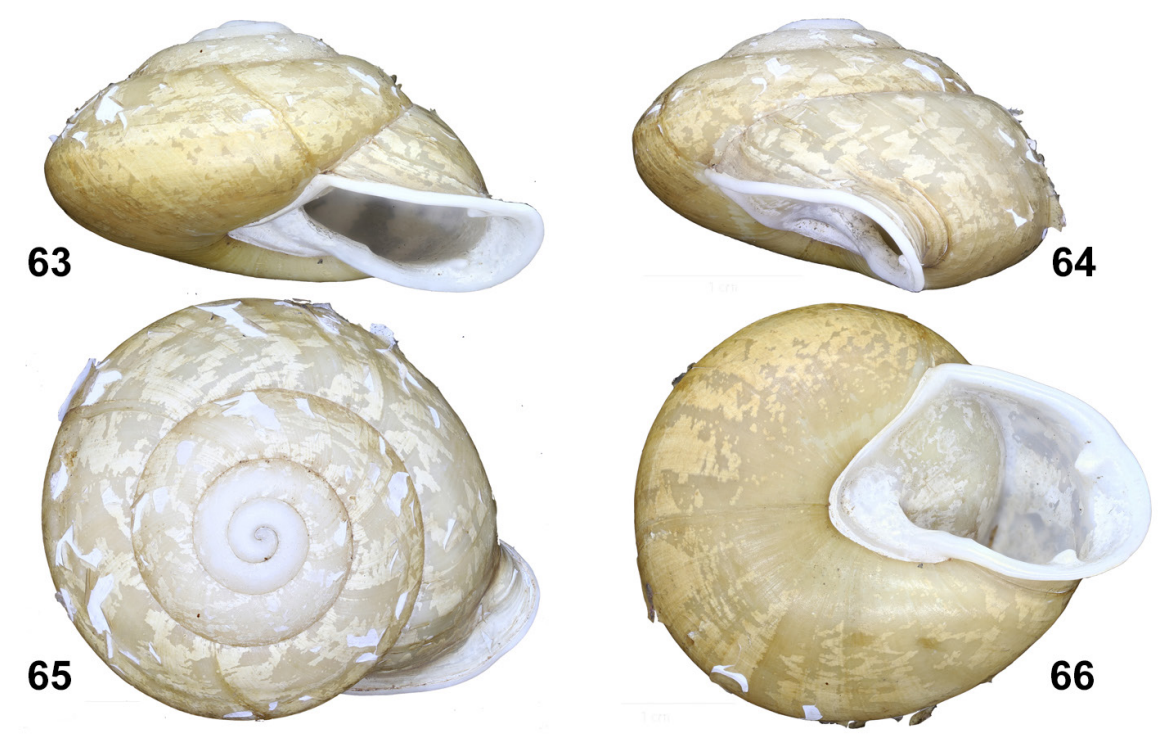

67

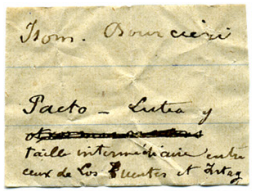

68

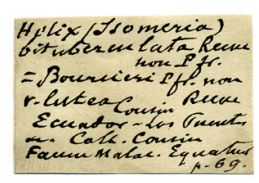

Figs 63-68. Isomeria bourcieri (L. Pfeiffer, 1853), syntype of Isomeria bourcieri var. lutea Cousin, 1887 RBINS MT.3817 (D $31.8 \mathrm{~mm}$ ): 63 - apertural view, 64 - right lateral view, 65 - apical view, 66 - umbilical view, 67 - label by Cousin, 68 label by Dautzenberg 

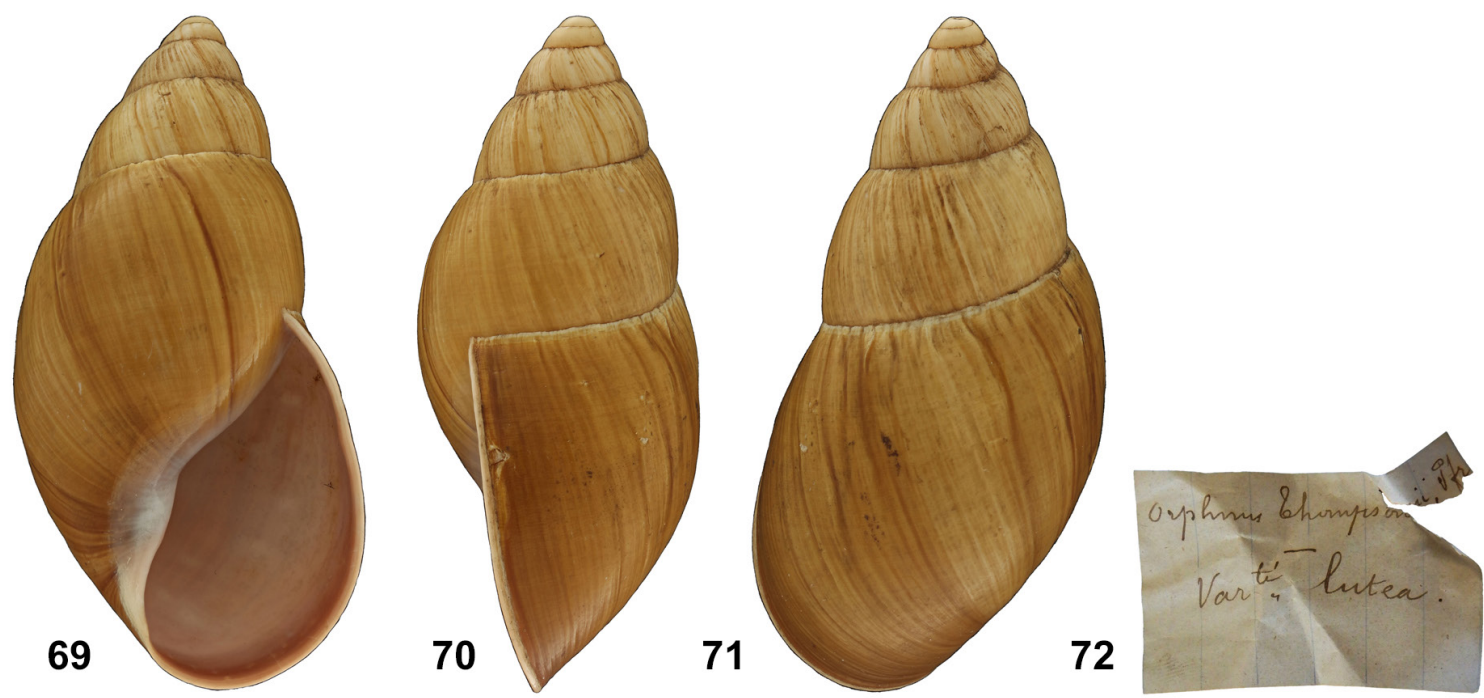

Figs 69-72. Kara thompsonii (L. Pfeiffer, 1845), lectotype of Orphnus thompsoni var. lutea Cousin, 1887 RBINS MT.2358 (H $75.6 \mathrm{~mm}$ ): 69 - apertural view, 70 - right lateral view, 71 - dorsal view, 72 - label by Dautzenberg

Type evidence. The material is ex Dautzenberg ex Cousin collection; label written in Dautzenberg's handwriting.

Remarks. In the handwritten catalogue Chilguiltina is among the 14 localities where this variety was found, but apparently Cousin only mentioned the three best-known localities in his publication. Chilguiltina could not be found with modern gazetteers. The specimens have lost their original colour. Current systematic position. Drymaeus (Drymaeus) nystianus (L. Pfeiffer, 1853) (BREURE 2011).

\section{lyzarzaburui, Ammonoceras Jousseaume, 1887 [Scolodontidae]}

Figs 75-80
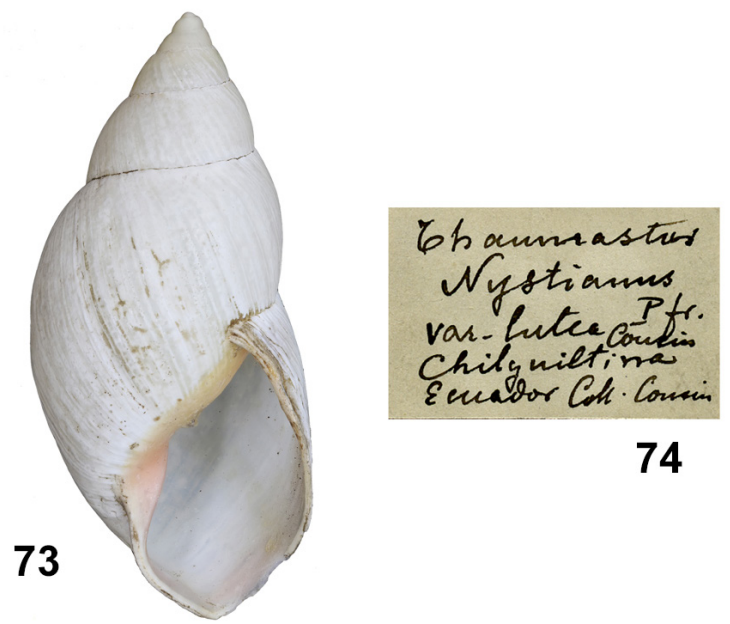

74

Figs 73-74. Drymaeus (Drymaeus) nystianus (L. Pfeiffer, 1853), possible syntype of Thaumastus (?) nystianus var. lutea Cousin, 1887 RBINS MT.3818 (H $36.1 \mathrm{~mm})$ : 73 apertural view, 74 - label by Dautzenberg

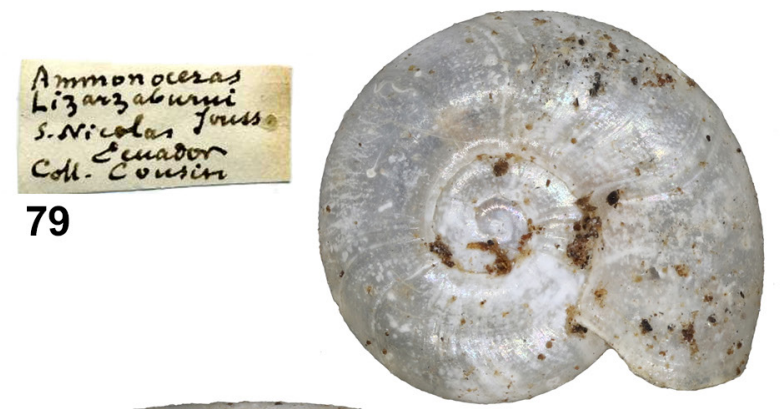

75

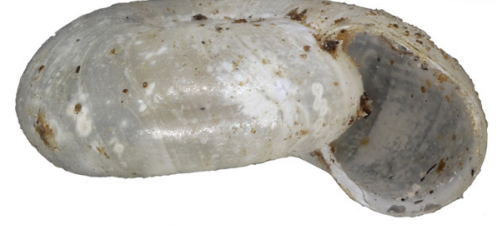

76

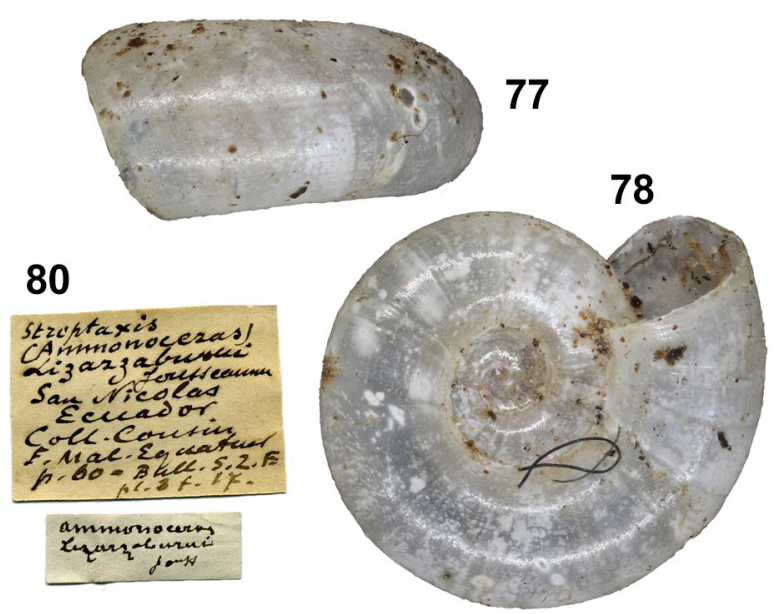

Figs 75-80. Drepanostomella lyzarzaburui (Jousseaume, 1887), syntype RBINS MT.3836 (D 2.4 mm): 75 - apical view, 76 - apertural view, 77 - right lateral view, 78 - umbilical view, 79 - label by Dautzenberg, 80 - labels by Dautzenberg and presumably by Jousseaume 
Ammonoceras lyzarzaburui JoUSSEAUME 1887: 174, pl. 3 fig. 17.

Type locality. "San-Nicolas, canton de Mégia".

Dimensions. "gr. diam $2^{\mathrm{mm}} 5$; p. diam., $2^{\mathrm{mm}}$; haut. $1^{\mathrm{mm}}$; figured specimen D $2.4 \mathrm{~mm}$.

Type material. Syntypes RBINS MT.3836 (3).

Label. "amonoceras / lyzarzaburi / San N".

Type evidence. The material is ex Dautzenberg ex Cousin collection; one label presumably written in Jousseaume's handwriting, the two others in the hand of Dautzenberg.

Remarks. Additional type material is in the Paris museum: MNHN-IM-2000-25754 (2), MNHNIM-2000-25755 (4), syntypes.

Current systematic position. Drepanostomella lyzarzaburui (Jousseaume, 1887).

\section{major, Hamadryas fallax Cousin, 1887 [Bulimulidae]}

Figs $81-85$

Hamadryas fallax var. major COUSIN 1887: 219.
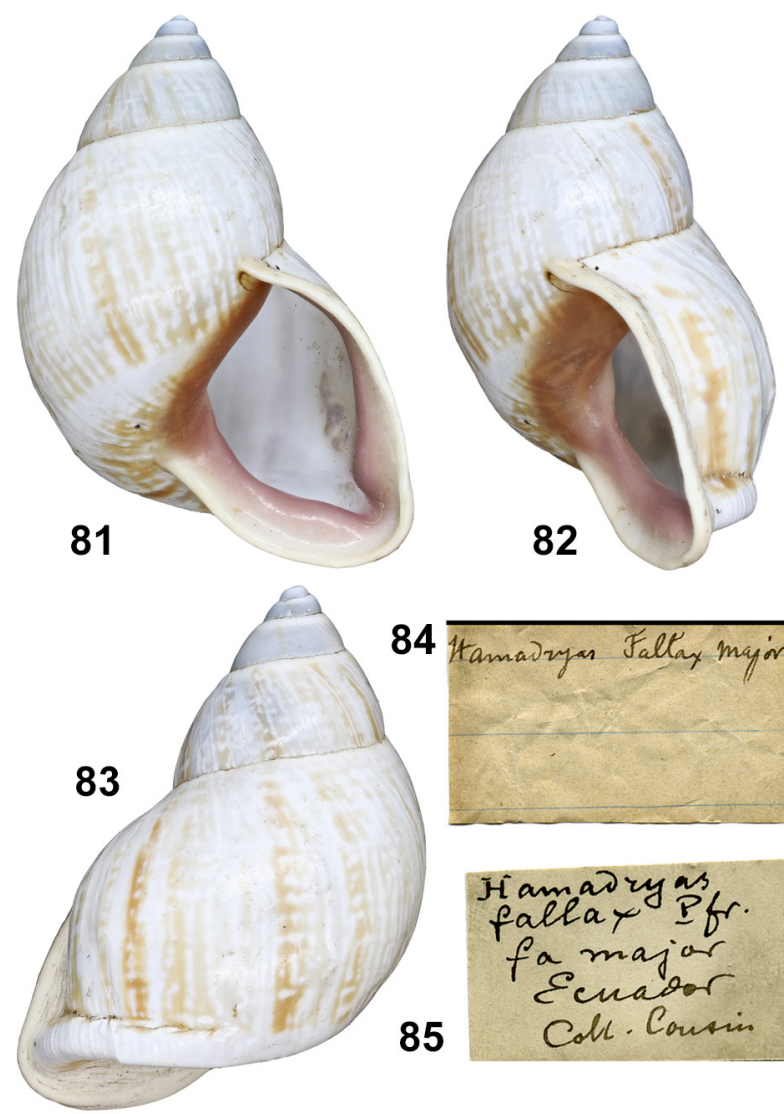

84

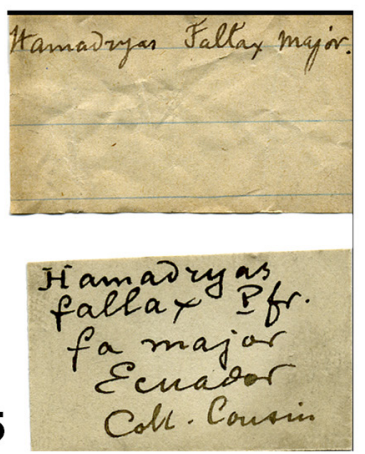

Figs 81-85. Drymaeus (Drymaeus) fallax (L. Pfeiffer, 1853), syntype of Hamadryas fallax var. major Cousin, 1887 RBINS MT.3815 (H 24.2 mm): 81 - apertural view, 82 right lateral view, 83 - dorsal view, 84 - label by Cousin, 85 - label by Dautzenberg
Type locality. "le long du chemin d'Aloag à Chones, canton de Mégia" [along the road from Aloag to Chones, canton of Mégia].

Dimensions. Not given; figured specimen $\mathrm{H}$ $24.2 \mathrm{~mm}$.

Type material. RBINS MT.3815 (1), syntype.

Label. "Ecuador".

Type evidence. The material is ex Dautzenberg ex Cousin collection; taxon label written in Cousin's handwriting, plus a label in Dautzenberg's hand.

Current systematic position. Drymaeus (Drymaeus) fallax (L. Pfeiffer, 1853) (BREURE 2011).

\section{marianita, Neritostoma Cousin, 1887 [Succineidae]}

Figs 86-90

Neritostoma marianita COUSIN 1887: 236, pl. 4 figs 5-5bis.

Type locality. "San-Nicolas".

Dimensions. "long., $6^{\mathrm{mm}}$; gr. diam., $4^{\mathrm{mm}}$; pet.diam. $3^{\mathrm{mm}}$ "; figured specimen $\mathrm{H} 6.6 \mathrm{~mm}$.

Type material. RBINS MT.3837 (15), syntypes.

Label. "neritostoma / marianita / sn. nicolas".
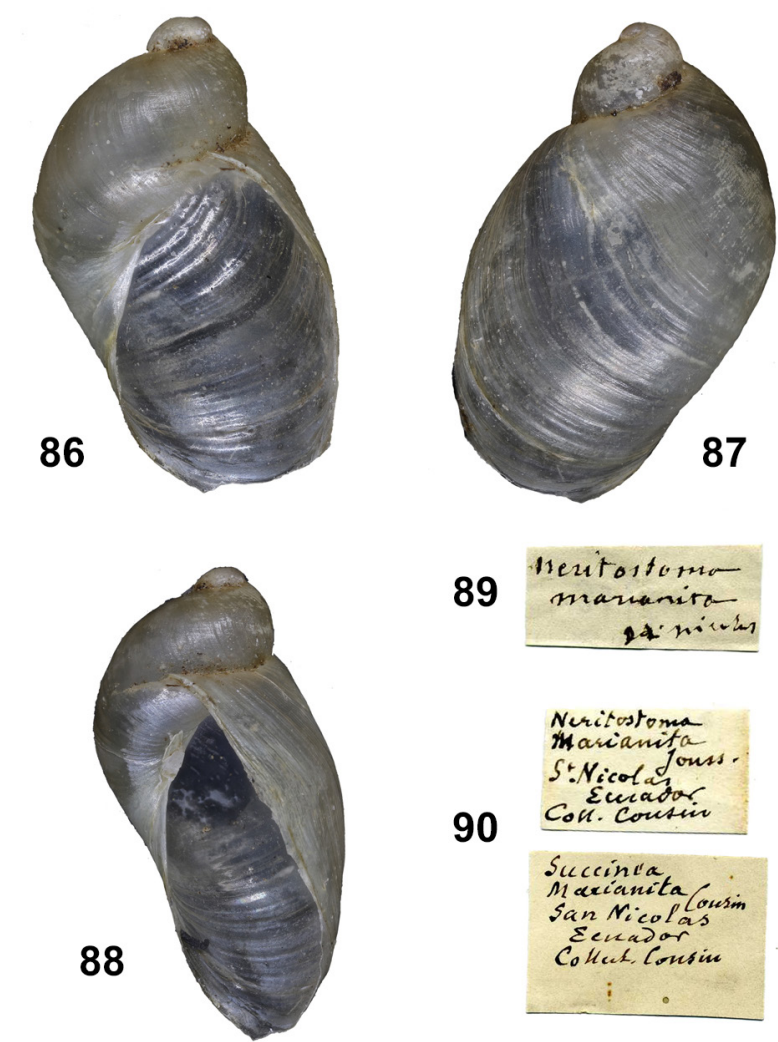

89
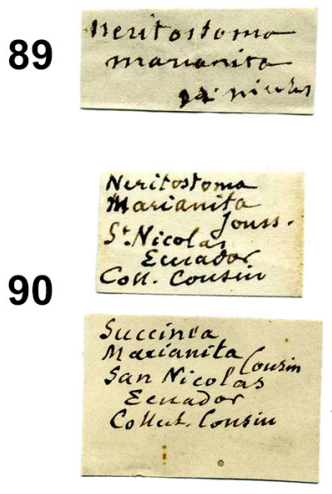

Figs 86-90. Succinea marianita (Cousin, 1887), syntype RBINS MT.3837 (H 6.6 mm): 86 - apertural view, 87 - dorsal view, 88 - right lateral view, 89 - label presumably by Jousseaume, 90 - labels by Dautzenberg 
Type evidence. The material is ex Dautzenberg ex Cousin collection; one label presumably written in Jousseaume's handwriting.

Remarks. The taxon has not been revised in later literature.

Current systematic position. Unknown, provisionally Succinea marianita (Cousin, 1887).

martinida, Guestieria Jousseaume, 1887 [Scolodontidae]

Figs 91-95

Guestieria martinida JOUSSEAUME 1887: 176, pl. 3 fig. 19.

Type locality. "San-Nicolas, canton de Mégia, province de Pichincha".

Dimensions. "gr. diam., $2^{\mathrm{mm}} 5$; p. diam., $2^{\mathrm{mm}}$; haut., $0^{\mathrm{mm}}$ 8"; figured specimen D $2.3 \mathrm{~mm}$.

Type material. RBINS MT.3830 (12), syntypes.

Label. "San Nicolas".

Type evidence. The material is ex Dautzenberg ex Cousin collection; label presumably written in Jousseaume's handwriting and additional labels in Dautzenberg's hand.

Remarks. More type material is in the Paris museum: MNHN-IM-2000-25756 (1), syntype.

Current systematic position. Guestieria martinida Jousseaume, 1887.

nigricans, Orphnus thompsoni Cousin, 1887 [Orthalicidae]

Figs 96-99

Orphnus thompsoni var. nigricans COUSIN 1887: 212.

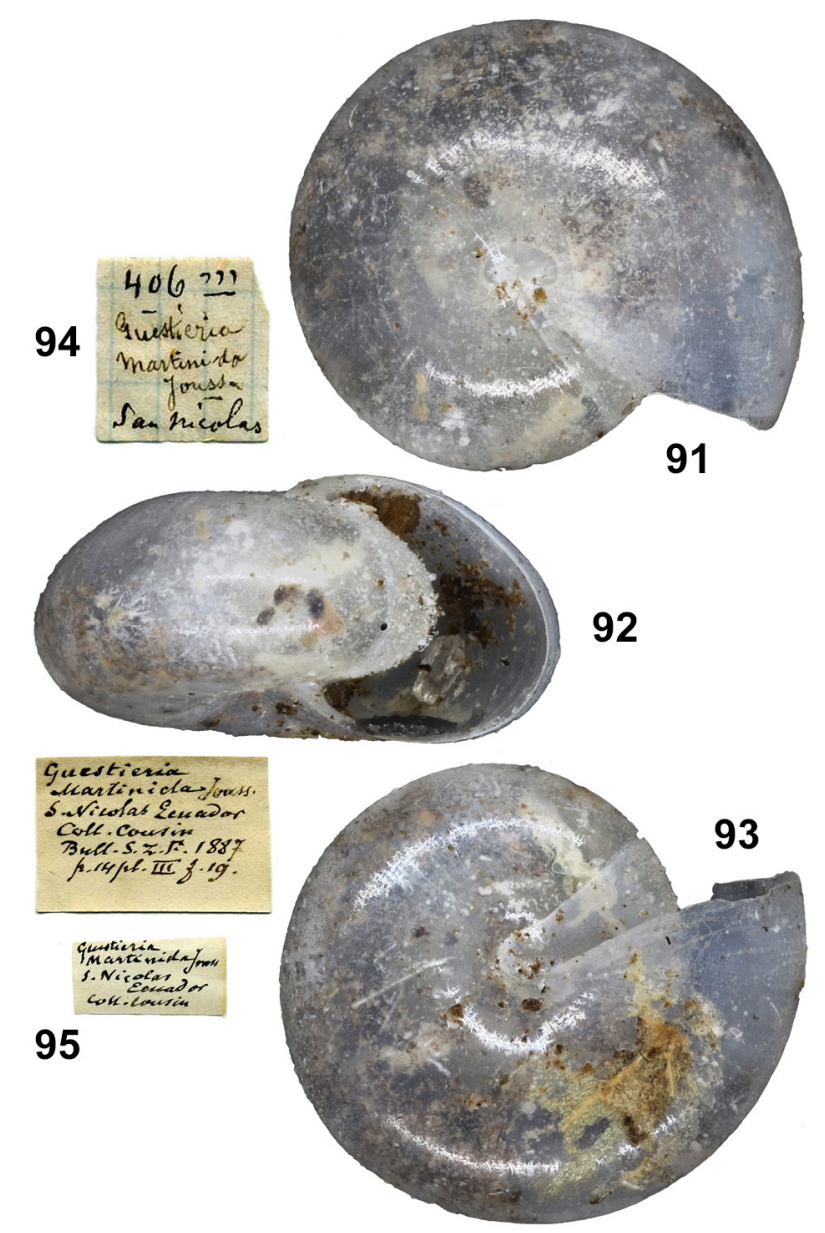

Figs 91-95. Guestieria martinida Jousseaume, 1887, syntype RBINS MT.3830 (D $2.2 \mathrm{~mm}$ ): 91 - apical view, 92 - apertural view, 93 - umbilical view, 94 - label by Jousseaume, 95 - labels by Dautzenberg

Type locality. "Cuença".
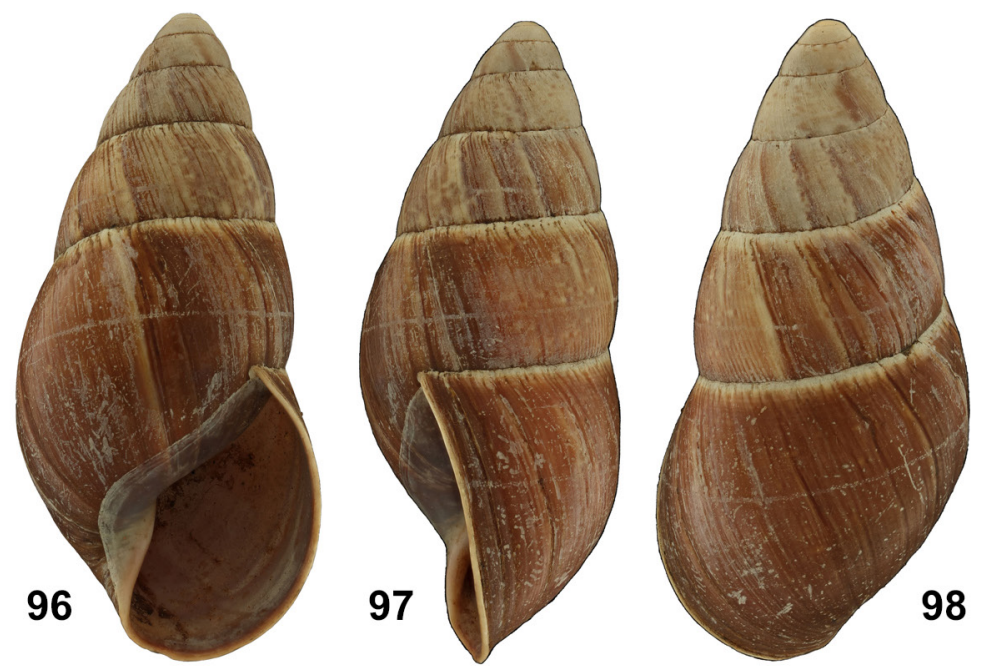

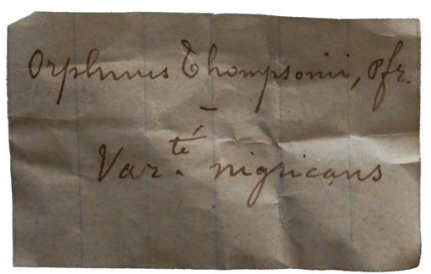

99

Figs 96-99. Kara thompsonii (L. Pfeiffer, 1845), lectotype of Orphnus thompsoni var. nigricans Cousin, 1887 RBINS MT.2363 (H $62.8 \mathrm{~mm}$ ): 96 - apertural view, 97 - right lateral view, 98 - dorsal view, 99 - label by Cousin 
Dimensions. Not given; figured specimen $\mathrm{H}$ $62.8 \mathrm{~mm}$.

Type material. RBINS MT.2363 (lectotype; BREURE 2011: 36); RBINS MT.2364 (3), paralectotypes.

Label. "Cuenca Ecuador".

Type evidence. The material is ex Dautzenberg ex Cousin collection; taxon label written by Cousin, locality label written in Dautzenberg's handwriting. Current systematic position. Kara thompsonii (L. Pfeiffer, 1845) (BREURE 2011).

\section{nigricans, Thaumastus (?) nystianus Cousin, 1887 [Bulimulidae]}

Figs 100-103

Thaumastus (?) nystianus var. nigricans COUSIN 1887: 220.

Type locality. "les bords du chemin qui conduit de Pomasqui à Chilguiltina" [the sides of the road that leads from Pomasqui to Chilguiltina].
Dimensions. Not given; figured specimen $\mathrm{H}$ $36.6 \mathrm{~mm}$.

Type material. RBINS MT.2365 (13), syntypes.

Label. "chemin de Pomasqui à Chiguiltina".

Type evidence. The material is ex Dautzenberg ex Cousin collection; label written in Dautzenberg's handwriting.

Current systematic position. Drymaeus (Drymaeus) nystianus (L. Pfeiffer, 1853) (BREURE 2011).

\section{olivacea, Orphnus thompsoni Cousin, 1887 [Orthalicidae]}

Figs 104-108

Orphnus thompsoni var. olivacea COUSIN 1887: 212.

Type locality. "Cuença".

Dimensions. Not given; figured specimen $\mathrm{H}$ $69.0 \mathrm{~mm}$.

Type material. RBINS MT.2366 (lectotype; BREURE 2011: 36); RBINS MT.2367 (3), paralectotypes.
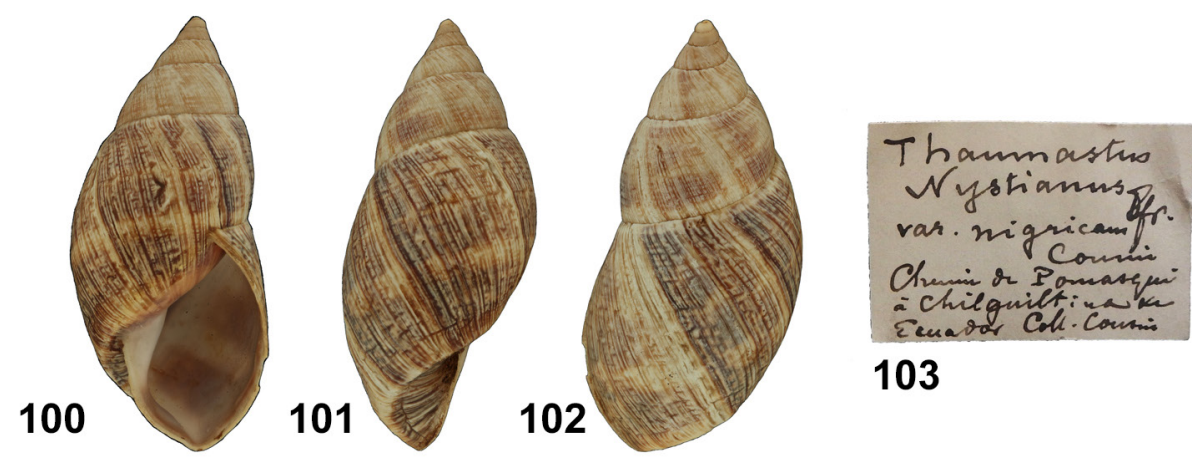

103

Figs 100-103. Drymaeus (Drymaeus) nystianus (L. Pfeiffer, 1853), syntype of Thaumastus (?) nystianus var. nigricans Cousin, 1887 RBINS MT.2365 (H 36.6 mm): 100 - apertural view, 101 - left lateral view, 102 - dorsal view, 103 - label by Dautzenberg
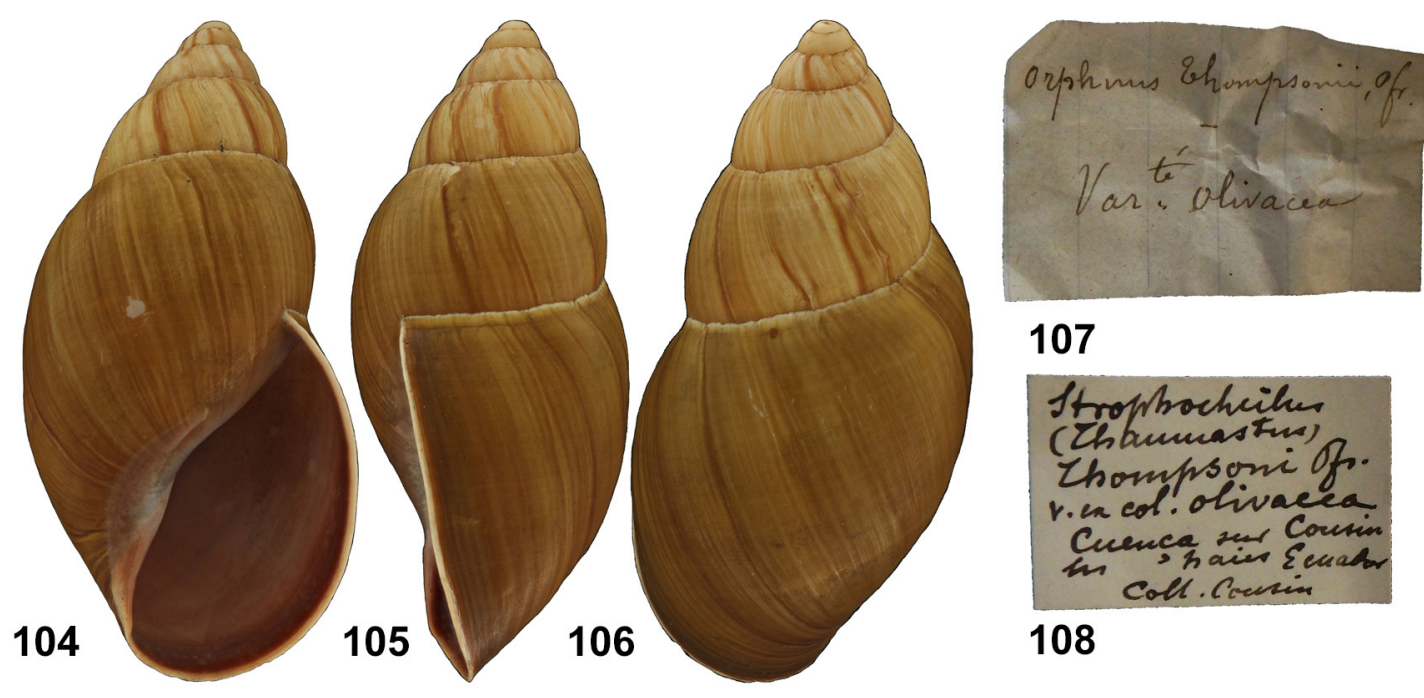

107

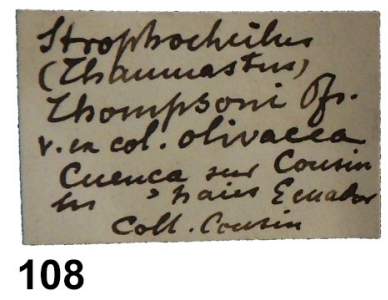

Figs 104-108. Kara thompsonii (L. Pfeiffer, 1845), lectotype of Orphnus thompsoni var. olivacea Cousin, 1887 RBINS MT.2366 (H 69.0 mm): 104 - apertural view, 105 - right lateral view, 106 - dorsal view, 107 - label by Cousin, 108 - label by Dautzenberg 
Label. "Cuenca Ecuador".

Type evidence. The material is ex Dautzenberg ex Cousin collection; taxon label written by Cousin, locality label written in Dautzenberg's handwriting. Current systematic position. Kara thompsonii (L. Pfeiffer, 1845) (BREURE 2011).

\section{puntaplaya, Ampullaria Cousin, 1887 [Ampulariidae]}

Figs 109-113

Ampullaria puntaplaya COUSIN 1887: 278, pl. 4 fig. 2.

Type locality. "Puntaplaya".

Dimensions. "long., $37^{\mathrm{mm}}$; gr. diam., $35^{\mathrm{mm}}$; p. diam., $27^{\mathrm{mm}}$; ouverture, long., $26^{\mathrm{mm}}$; larg., $27^{\mathrm{mm}}$; figured specimen $\mathrm{H} 27.7 \mathrm{~mm}$.

Type material. RBINS MT.3849 (8), paralectotypes.

Label. "Puntaplaya".

Type evidence. The material is ex Dautzenberg ex Cousin collection; label in Cousin's handwriting.

Remarks. Additional type material in Paris museum: MNHN-IM-2000-4622 (lectotype; COWIE \& HÉROS 2012: 816); MNHN-IM-2000-4621, paralectotype.

Current systematic position. Pomacea (Pomacea) puntaplaya (Cousin, 1887).

\section{raphaelis, Limnaea Jousseaume, 1887}

\section{[Lymnaeidae]}

Figs 114-118

Limnaea raphaelis JOUSSEAUME 1887: 183, pl. 3 fig. 1.

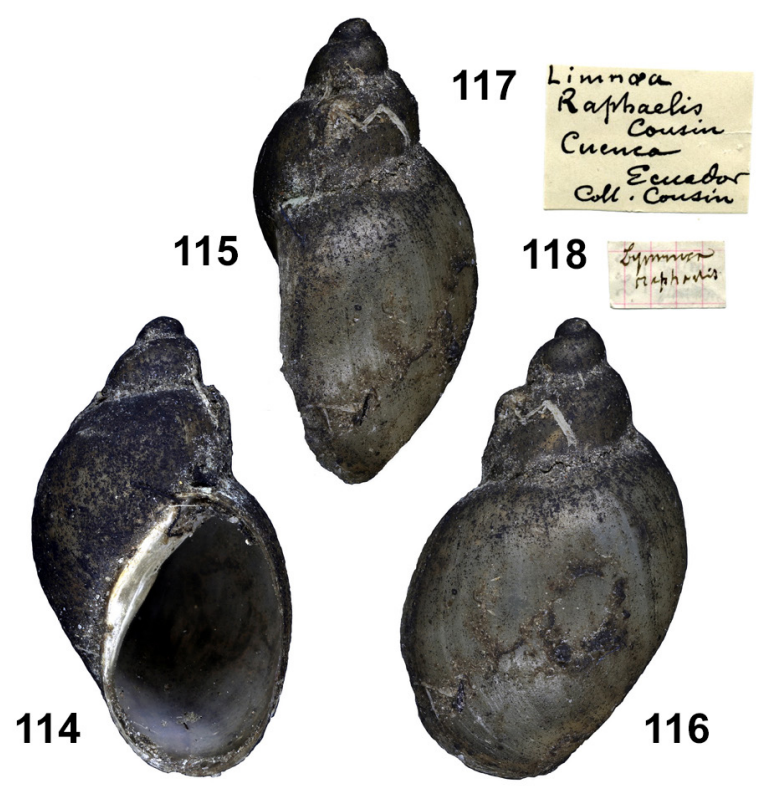

Figs 114-118. Lymnaea raphaelis (Jousseaume, 1887), syntype RBINS MT.3828 (H 7.0 mm): 114 - apertural view, 115 - right lateral view, 116 - dorsal view, 117 - label by Dautzenberg, 118 - label presumably by Jousseaume

Type locality. "à environs 2570 mètres (...) la région sud de Cuenca (prov. d'Azuay)" [at about 2570 meters (...) the southern region of Cuenca (prov. of Azuay)].

Dimensions. "long., 7 à 9mm; gr. diam., 6 à $4^{\mathrm{mm}}$; p. diam., 4 à $3^{\mathrm{mm}}$; ouverture, long., 5 à $3^{\mathrm{mm}} 5$; larg., 3 à $2^{\mathrm{mm}}$ 5"; figured specimen H $7.0 \mathrm{~mm}$.
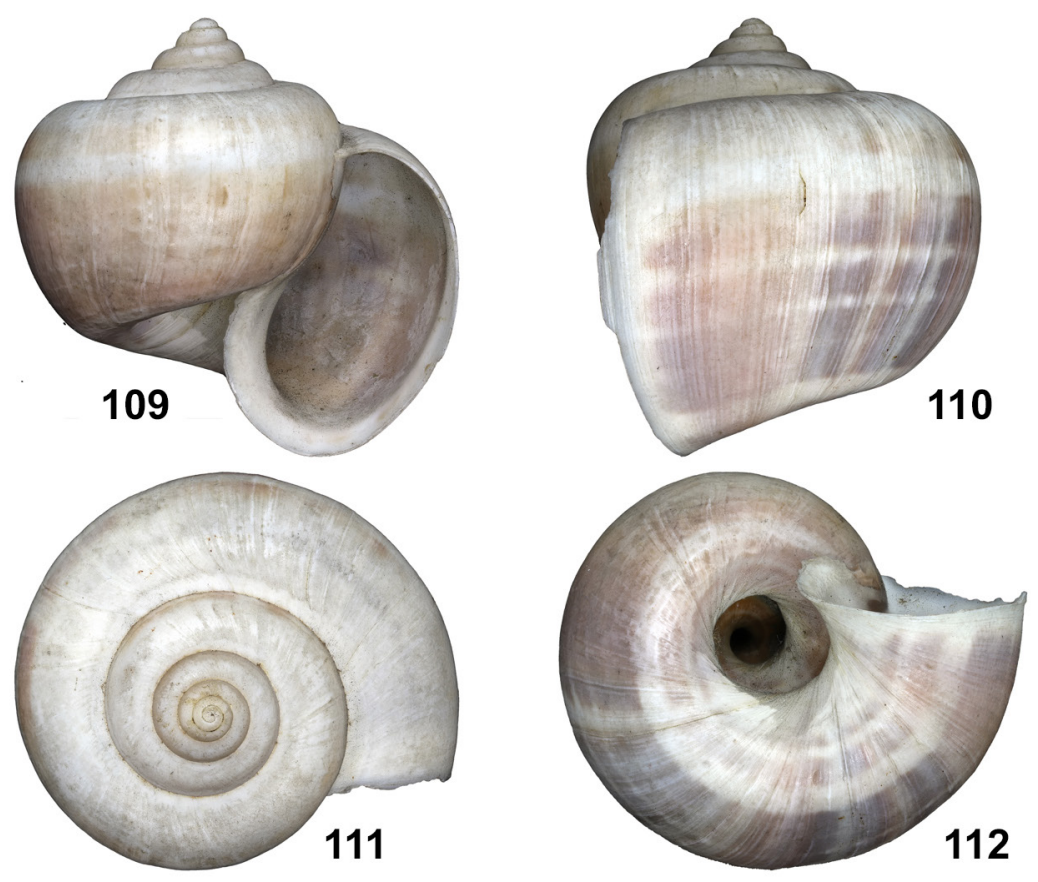

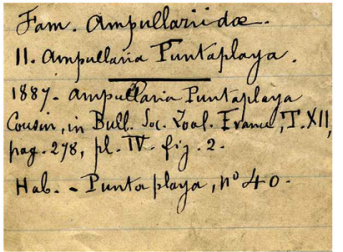

113

Figs 109-113. Pomacea (Pomacea) puntaplaya (Cousin, 1887), paralectotype RBINS MT.3849 (H 27.7 mm): 109 - apertural view, 110 - right lateral view, 111 - apical view, 112 - umbilical view, 113 - label by Cousin 
Type material. RBINS MT.3827 (23), MT.3828 (112), syntypes.

Label. "Lymnaea / raphaelis // Cuenca", "Cuenca", "près Pungavo".

Type evidence. The material is ex Dautzenberg ex Cousin collection; one label presumably in Jousseaume's hand, two other labels both in handwriting of Dautzenberg.

Remarks. The locality 'Pungavo' could not be located with modern gazetteers; it is possibly a misspelling. The taxon has not been revised in recent literature. Current systematic position. Unknown, provisionally Lymnaea raphaelis (Jousseaume, 1887).

\section{reyrei, Ampullaria Cousin, 1887}

\section{[Ampullariidae]}

Figs 119-123

Ampullaria reyrei COUSIN 1887: 279, pl. 4 fig. 7.

Type locality. "Napo".

Dimensions. "long., $41^{\mathrm{mm}}$; grand diamètre, $33^{\mathrm{mm}}$; petit diamètre, $16^{\mathrm{mm}}$; ouverture, long., $29^{\mathrm{mm}}$; larg., $16^{\mathrm{mm}}$; figured specimen H $37.7 \mathrm{~mm}$.

Type material. RBINS MT.1153 (1), syntype. Label. "Napo Ecuador".

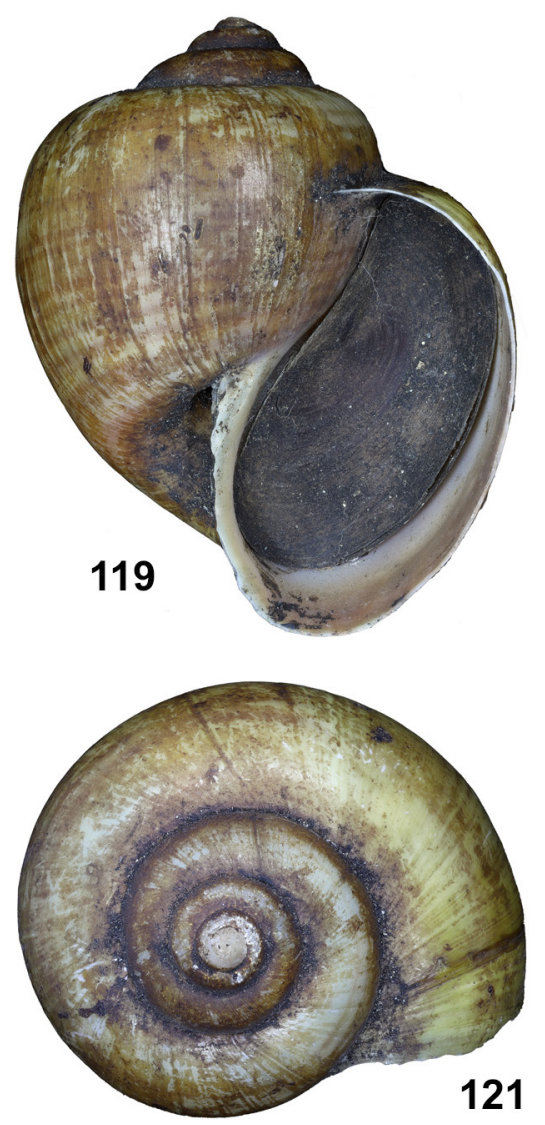

Type evidence. The material is ex Dautzenberg ex Cousin collection; label written in Dautzenberg's handwriting.

Remarks. Additional type material in Paris museum: MNHN-IM-2000-23087 (1), probable syntype (see COWIE \& HÉROS 2012: 816).

Current systematic position. Pomacea (Pomacea) reyrei (Cousin, 1887).

tenuissima, Ampullaria Jousseaume, 1894

[Ampulariidae]

Figs 124-130

Ampullaria tenuissima JoUSSEAUME 1894: 120, fig.

Type locality. "Équateur".

Dimensions. "longueur, 67 millimètres; largeur, 50 millimètres; épaisseur, 42 millimètres; ouverture: longueur, 50 millimètres; largeur, 28 millimètres"; figured specimen $\mathrm{H} 60.7 \mathrm{~mm}$.

Type material. RBINS MT.3850 (5), syntypes.

Label. "Aguarico".

Type evidence. The material is ex Dautzenberg ex Cousin collection; three labels written in the hand of Jousseaume, one other written in Dautzenberg's handwriting.
120

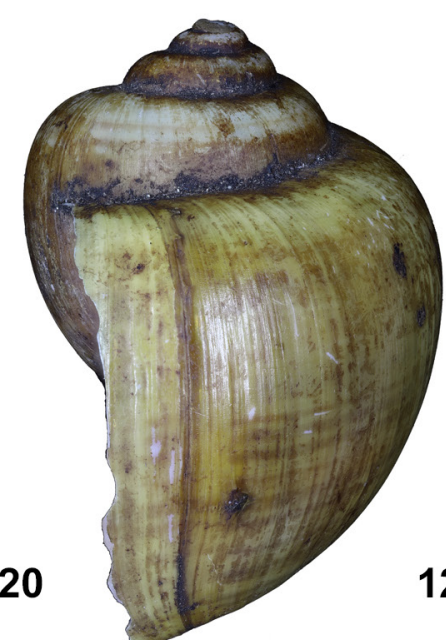

123
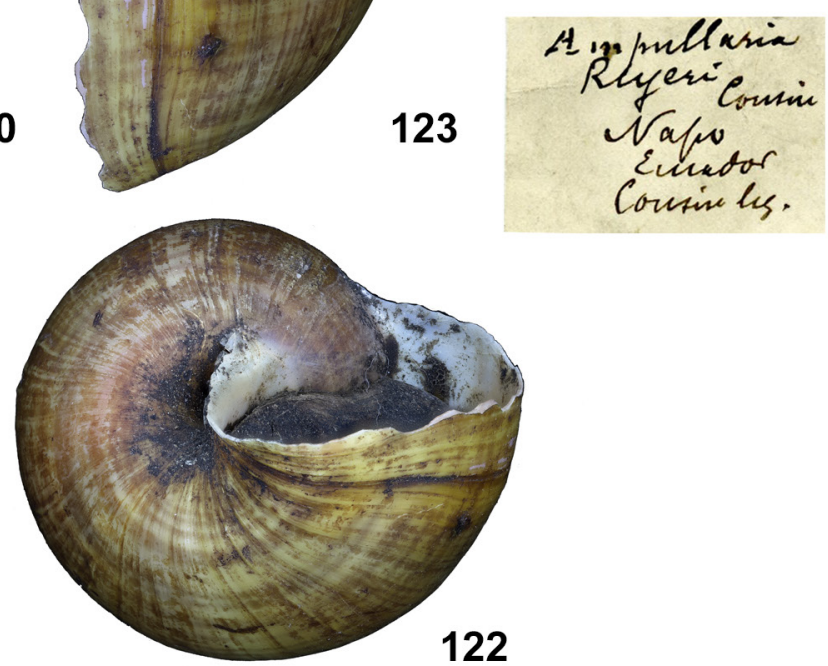

Figs 119-123. Pomacea (Pomacea) reyrei (Cousin, 1887), syntype RBINS MT.1153 (H 37.7 mm): 119 - apertural view, 120 right lateral view, 121 - apical view, 122 - umbilical view, 123 - label by Dautzenberg 

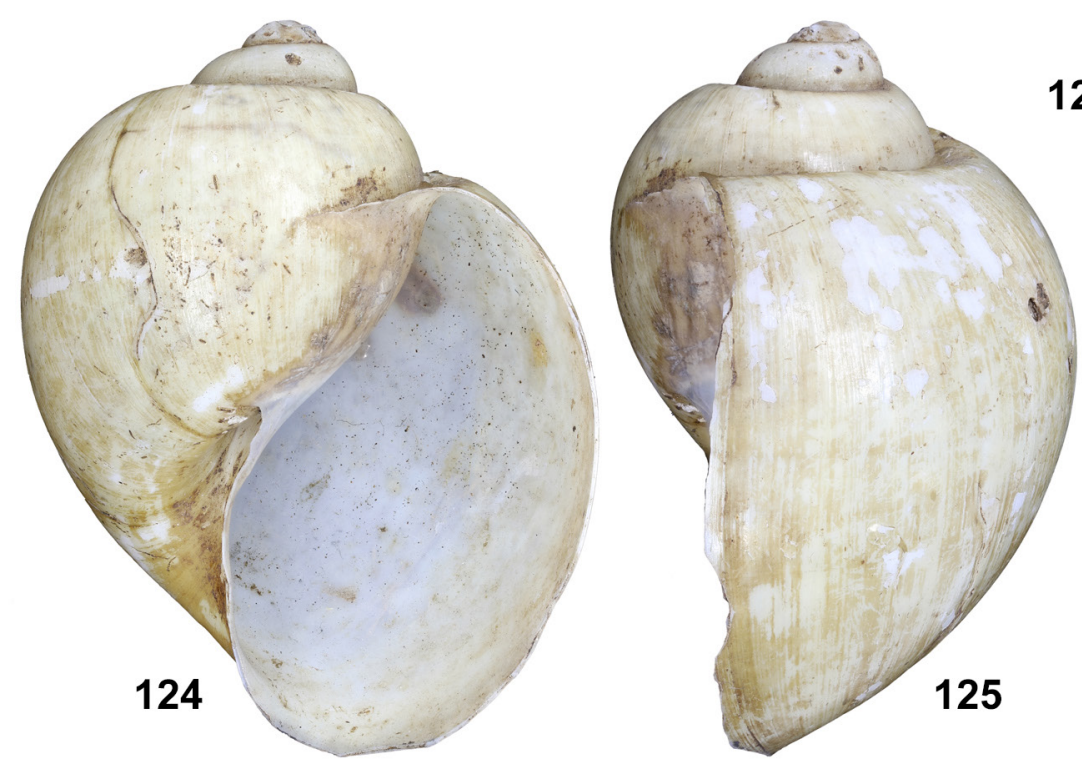

128

45. Oempontlen'd nowels

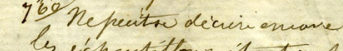

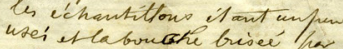
hlaces - dither 149 ge $188 \%$
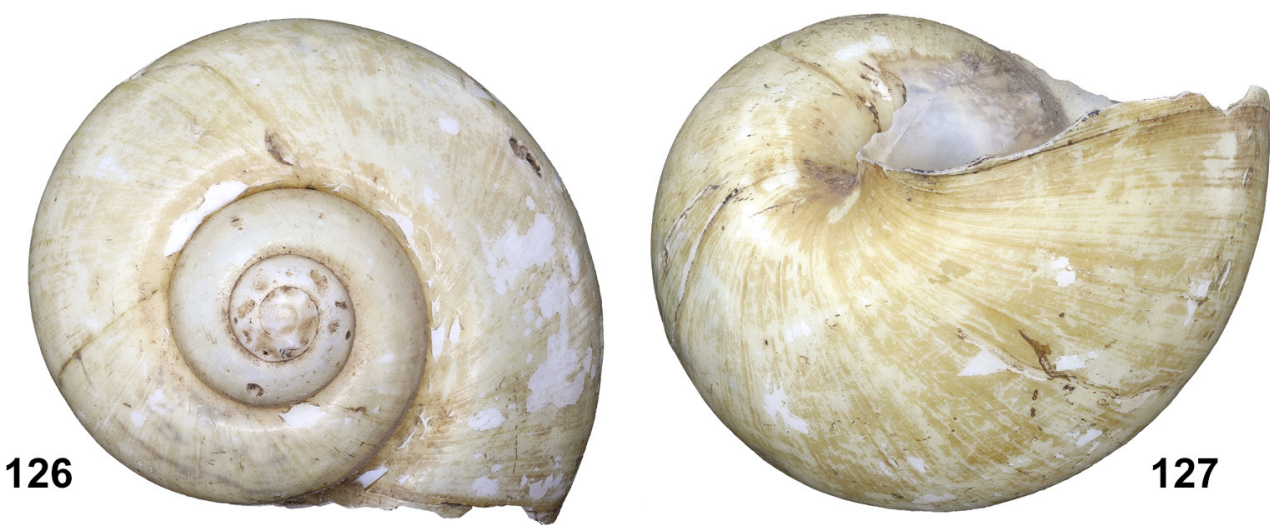

Figs 124-130. Pomacea (Pomacea) tenuissima (Jousseaume, 1894), syntype RBINS MT.3850 (H 60.7 mm): 124 - apertural view, 125 - right lateral view, 126 - apical view, 127 - umbilical view, 128 - label by Jousseaume, 129 - labels by Jousseaume, 130 - label by Dautzenberg

Remarks. Jousseaume explicitly mentioned his species was based on material from Cousin. One of the specimens closely matches the original figure. COWIE \& THIENGO (2004: 76) considered this taxon as a valid species.

Current systematic position. Pomacea (Pomacea) tenuissima (Jousseaume, 1894).

\section{valenzuela, Leptinaria Jousseaume, 1887} [Achatinidae]

Figs 131-133

Leptinaria valenzuela JOUSSEAUME 1887: 170, pl. 3 fig. 4.

Type locality. "la Coca, province d'Oriente". Dimensions. "long., $10^{\mathrm{mm}}$; larg., $4^{\mathrm{mm}} 5$; ouverture: long., $4^{\mathrm{mm}}$; larg., $3^{\mathrm{mm}}$; figured specimen H $9.3 \mathrm{~mm}$. Type material. RBINS MT.3846 (60), syntypes. Label. "La Coca ? Prov. Oriente".
Type evidence. The material is ex Dautzenberg ex Cousin collection; label written in Cousin's handwriting.
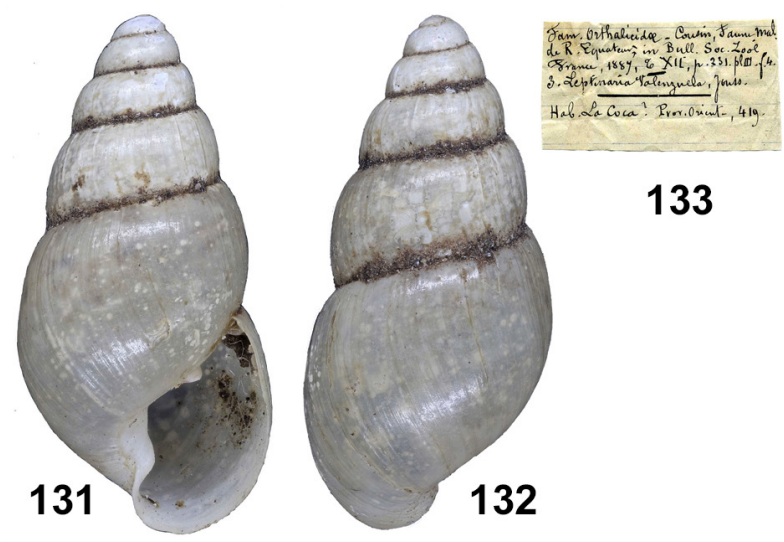

Figs 131-133. Leptinaria unilamellata (d'Orbigny, 1835), syntype of Leptinaria valenzuela Jousseaume, 1887 RBINS MT.3846 (H $9.3 \mathrm{~mm}$ ): 131 - apertural view, 132 - dorsal view, 133 - label by Cousin 
Remarks. The locality given by Jousseaume is currently named Puerto Franscico de Orrealana.

Current systematic position. Leptinaria unilamellata (d'Orbigny, 1835) (F. NAGGS, pers. commun.).

\section{vasconesi, Cyclophorus Jousseaume, 1897 [Neocyclotidae]}

Figs 134-139

Cyclophorus vasconesi JOUSSEAUME 1897: 250, fig.

Type locality. "Le Coca".

Dimensions. "Diam. maj. 29 mm. min. 25 mm. alt. $15 \mathrm{~mm}$ "; figured specimen D $15.5 \mathrm{~mm}$.

Type material. RBINS MT.3829 (1), MT.3838 (1), possible syntypes.

Labels. "Machaij (occident de Pujili)" [Machaij (west of Pujili)], "Puca Jacu, Lampadora cerca Quevedo".
Type evidence. Material is marked ex Dautzenberg ex Cousin collection, labels in handwriting Jousseaume. Remarks. The locality given by Jousseaume is currently named Puerto Franscico de Orrealana. Jousseaume explicitly stated his species was based on material from the Cousin collection. Since the labels do not fully match, the material is considered as possible syntypes.

Current systematic position. Lagocyclus vasconesi (Jousseaume, 1897).

\section{zebra, Orphnus thompsoni Cousin, 1887} [Orthalicidae]

Figs 140-143

Orphnus thompsoni var. zebra COUSIN 1887: 212.
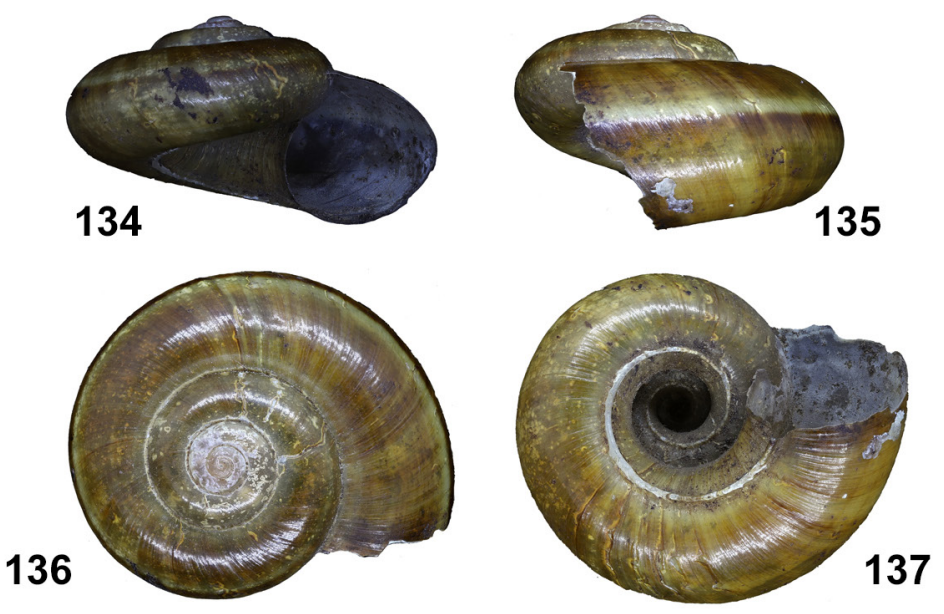

138

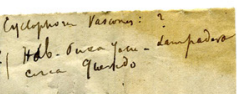

139

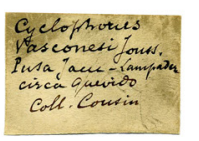

Figs 134-139. Lagocyclus vasconesi (Jousseaume, 1897), possible syntype RBINS MT.3829 (D 15.5 mm): 134 - apertural view, 135 - right lateral view, 136 - apical view, 137 - umbilical view, 138 - label by Jousseaume, 139 - label by Dautzenberg
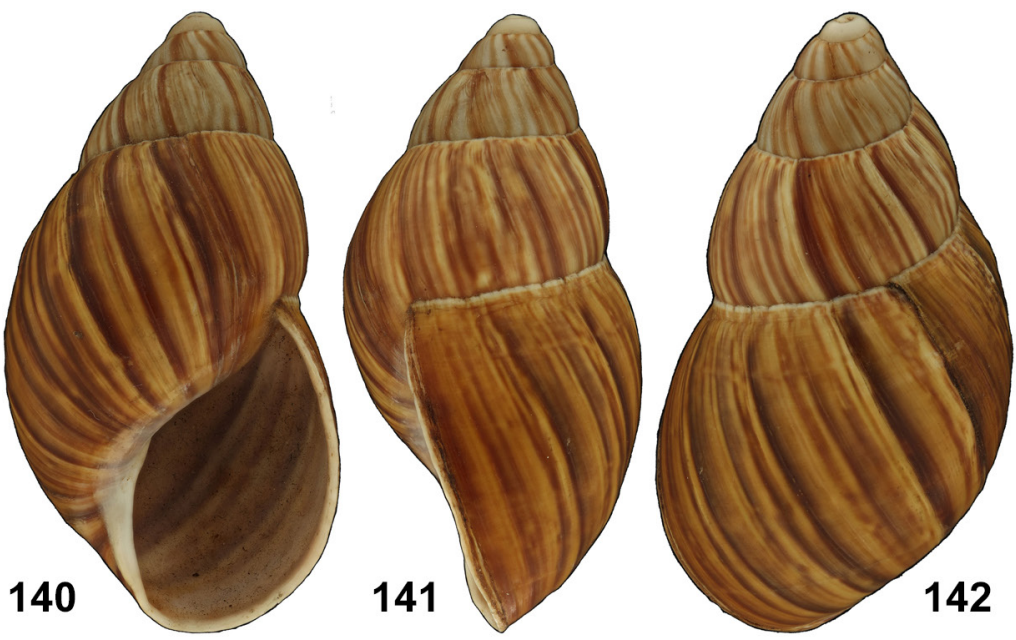

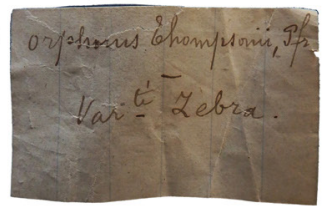

143

Figs 140-143. Kara thompsonii (L. Pfeiffer, 1845), lectotype of Orphnus thompsoni var. zebra Cousin, 1887 RBINS MT.2375

(H $75.0 \mathrm{~mm}$ ): 140 - apertural view, 141 - right lateral view, 142 - dorsal view, 143 - label by Cousin 
Type locality. "près Azagues [Azogues], sur la pente, à environs $2400 \mathrm{~m}$ alt." [near Azogues, on the slope, at about $2400 \mathrm{~m}$ altitude].

Dimensions. Not given; figured specimen $\mathrm{H}$ $49.0 \mathrm{~mm}$.

Type material. RBINS MT.2375 (lectotype; BREURE 2011: 42); RBINS MT.2376 (9), paralectotypes.

Label. "près Azagues sur la pente à envir. 2400m alt.".

Type evidence. The material is ex Dautzenberg ex Cousin collection; taxon label written by Cousin, locality label written in Dautzenberg's handwriting.

Current systematic position. Kara thompsonii (L. Pfeiffer, 1845) (BREURE 2011).

\section{REFERENCES}

ANONYMOUS 1883. Liste des membres de la Société au 1er Avril 1883. Bulletin de la Société zoologique de France 8: 7-15.

ANONYMOUS 1889a. Liste des membres de la Société au 22 Janvier 1889 avec la date de leur admission. Bulletin de la Société zoologique de France 14: 5-16.

ANONYMOUS 1889b. Séance du 12 Novembre 1889. Bulletin de la Société zoologique de France 14: 350-362.

ANONYMOUS 1891. Liste des membres de la Société au 27 Janvier 1889 avec la date de leur admission. Bulletin de la Société zoologique de France 16: 5-18.

ANONYMOUS 1897. Liste des membres de la Société au 31 Janvier 1897 avec la date de leur admission. Bulletin de la Société zoologique de France 22: 13-28.

ANONYMOUS 1900. Liste des membres décédés pendant l'année 1899. Bulletin de la Société zoologique de France 25: 26.

BreURE A. S. H. 1976. Types of Bulimulidae in the Muséum national d'Histoire naturelle, Paris. Bulletin du Muséum national d'Histoire naturelle, 3e série, 331, Zoologie 233: 1137-1187.

Breure A. S. H. 2011. Annotated type catalogue of the Orthalicoidea (Mollusca, Gastropoda) in the Royal Belgian Institute of Sciences, Brussels, with descriptions of two new species. Zookeys 101: 1-50. https:// doi.org/10.3897/zookeys.101.1133

Cousin A. 1887. Faune malacologique de la République de l'Équateur. Bulletin de la Société zoologique de France 12: $187-287$.

Cousin R. 2019. Dr Auguste Édouard Cousin. Available at https://www.myheritage.fr/person-3000047 $7238682172386821 /$ dr-augusteedouard-cousin-noel? show $=$ events (accessed 12 May 2019).

CowIE R. H., HÉros V. 2012. Annotated catalogue of the types of Ampullariidae (Mollusca, Gastropoda) in the Muséum national d'Histoire naturelle, Paris, with lectotype designations. Zoosystema 34(4): 793-824. https://doi.org/10.5252/z2012n4a8

\section{ACKNOWLEDGEMENTS}

The support of Yves BARETTE, AURORE CORDIER, YVES SAMYN and FLORENCE TRUS with the preparation of this manuscript is gratefully acknowledged. The first and last are credited for the photographs. FRED NAGGS was kind enough to give his opinion on Achatinidae. VIRGINIE HÉROs kindly supplied information not available on the website. JONATHAN ABLETT and three anonymous reviewers are thankfully acknowledged for their comments on a previous version of this manuscript.

COWIE R. H., THIENGO S. C. 2004. The apple snails of the Americas (Mollusca: Gastropoda: Ampullariidae: Asolene, Felipponea, Marisa, Pomacea, Pomella): A nomenclatural and type catalog. Malacologia 45: 41-100.

GEMERT L. J. VAN 2007. Een voorlopige bibliografie van Félix Jousseaume (1835-1921). A preliminary bibliography of Félix Jousseaume (1835-1921). De Kreukel 43 (6): 81-95.

GEMERT L. J. VAN, BREURE A. S. H. 2017. The tautonyms of Jousseaume: a taxonomical study. Folia Conchyliologica 42: 14-23.

JOUSSEAUME F. 1884. Description de mollusques nouveaux. Bulletin de la Société zoologique de France 9: 169-192.

Jousseaume F. 1887. Mollusques nouveaux de la République de l'Équateur. Bulletin de la Société zoologique de France 12: 165-186.

JOUSSEAUME F. 1894. Description d'un mollusque nouveau. Le Naturaliste (2)8: 120-121.

JOUSSEAUME F. 1897. Description d'une coquille nouveau. Le Naturaliste (2) 11: 250.

Jousseaume F. 1900. Mollusques terrestres. Clausilia, Rhodea et Bulimus Sud-Americanae. Bulletin de la Société philomatique de Paris 9: 5-44.

LAMY E., DAUTZENBERG P. 1952. Liste d'espèces décrites par le Dr. Jousseaume, d'après un manuscrit de feu A. Jousseaume. Journal de Conchyliologie 92 (2): 80-86.

MOLLUSCABASE 2019. Available at www.molluscabase.org (accessed 12 May 2019).

PARAENSE W. L. 1966. Biomphalaria amazonica and B. cousini, two new species of Neotropical planorbid molluscs. Revista Brasileira de Biología 26: 115-126.

PARAENSE W. L. 2004. Planorbidae, Lymnaeidae and Physidae of Ecuador (Mollusca: Basommatophora). Memorias del Instituto Oswaldo Cruz 99: 357-362. https://doi.org/10.1590/S0074-02762004000400003

Received: December 9th, 2019

Revised: January 22nd, 2020

Accepted: January 28th, 2020

Published on-line: March 16th, 2020 